Document downloaded from:

http://hdl.handle.net/10251/132135

This paper must be cited as:

Torregrosa, AJ.; Arnau Martínez, FJ.; Piqueras, P.; Sanchis-Pacheco, EJ.; Tartoussi, H. (2017). Phenomenological methodology for assessing the influence of flow conditions on the acoustic response of exhaust aftertreatment systems. Journal of Sound and Vibration. 396:289-306. https://doi.org/10.1016/j.jsv.2017.02.033

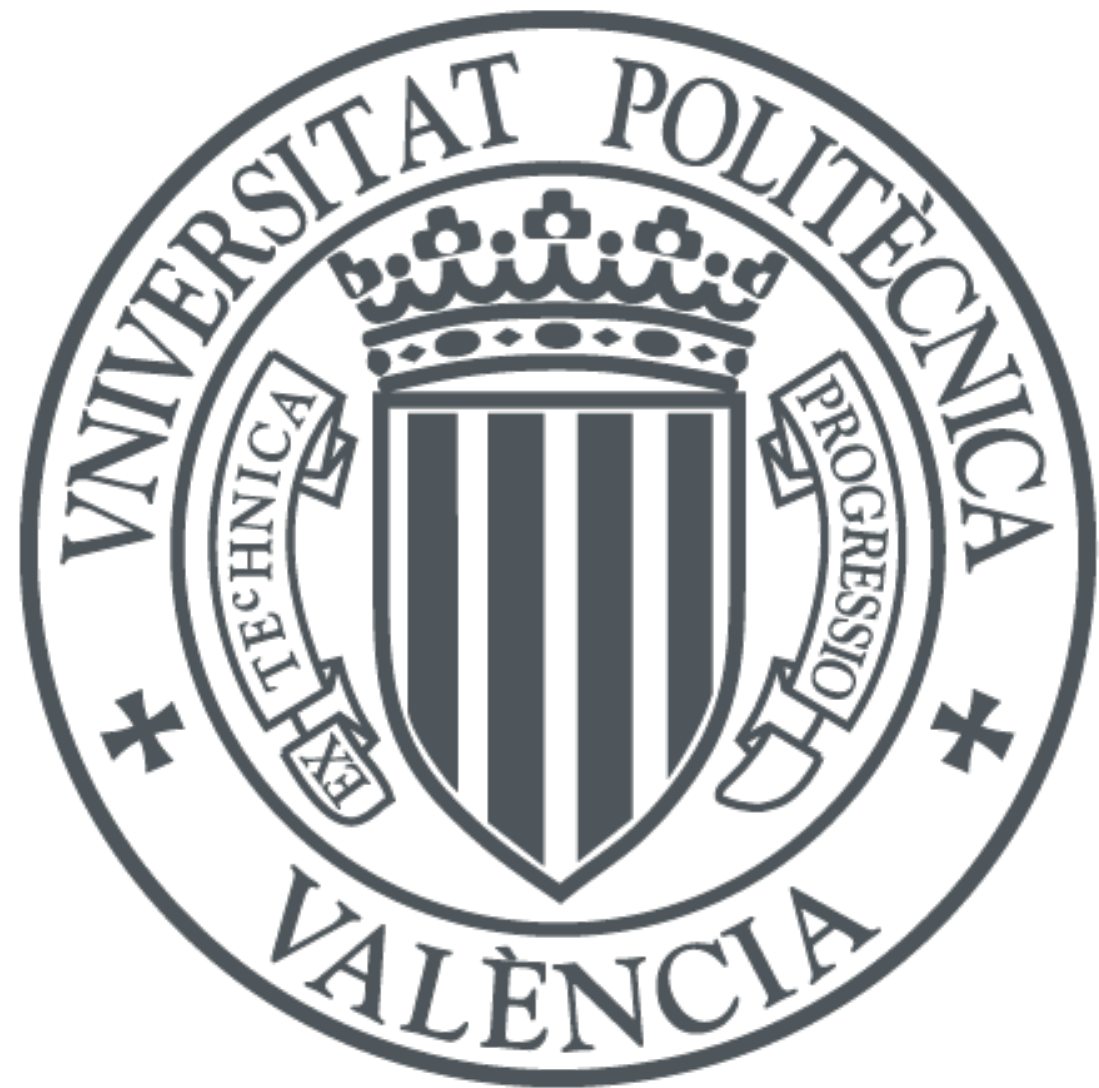

The final publication is available at

https://doi.org/10.1016/j.jsv.2017.02.033

Copyright Elsevier

Additional Information 


\title{
Phenomenological methodology for assessing the influence of flow conditions on the acoustic response of exhaust aftertreatment systems
}

\author{
A. J. Torregrosa ${ }^{\mathrm{a}}$, F. J. Arnau ${ }^{\mathrm{a}}$, P. Piqueras ${ }^{\mathrm{a}, *}$, E. J. Sanchis ${ }^{\mathrm{a}}$, H. Tartoussi ${ }^{\mathrm{b}}$ \\ ${ }^{a}$ CMT-Motores Térmicos, Universitat Politècnica de València, Camino de Vera s/n, 46022 Valencia, Spain. \\ ${ }^{b}$ Renault SA, 1 alle Cornuel, 91510 Lardy, France.
}

\begin{abstract}
The increasing limits of standards on aerosol and gaseous emissions from internal combustion engines have led to the progressive inclusion of different exhaust aftertreatment systems (EATS) as a part of the powertrain. Regulated emissions are generally abated making use of devices based on monolithic structures with different chemical functions. As a side effect, wave transmission across the device is affected and so is the boundary at the exhaust line inlet, so that the design of the latter is in turn affected. While some models are available for the prediction of these effects, the geometrical complexity of many devices makes still necessary in many cases to rely on experimental measurements, which cannot cover all the diversity of flow conditions under which these devices operate.

To overcome this limitation, a phenomenological methodology is proposed in this work that allows for the sound extrapolation of experimental results to flow conditions different from those used in the measurements. The transfer matrix is obtained from tests in an impulse rig for different excitation amplitudes and mean flows. The experimental coefficients of the transmission matrix of the device are fitted to Fourier series. It allows treating the influence of the flow conditions on the acoustic response, which is manifested on changes in the characteristic periods, separately from the specific properties of every device. In order to provide predictive capabilities to the method, the Fourier series approach is coupled to a gas dynamics model able to account for the sensitivity of propagation velocity to variations in the flow conditions.
\end{abstract}

Keywords: Aftertreatment system, acoustics, transfer matrix, test, modelling

\section{Nomenclature}
a Speed of sound
$a_{k} \quad$ Main Fourier series constant
$a_{k}^{\prime} \quad$ Residual Fourier series constant
A Cross-section area
$b_{k} \quad$ Main Fourier series constant
$b_{k}^{\prime} \quad$ Residual Fourier series constant

${ }^{*}$ Corresponding author. Tel.: +34 96 3877650, fax: +34 963877659.

Email address: pedpicab@mot.upv.es (P. Piqueras) 


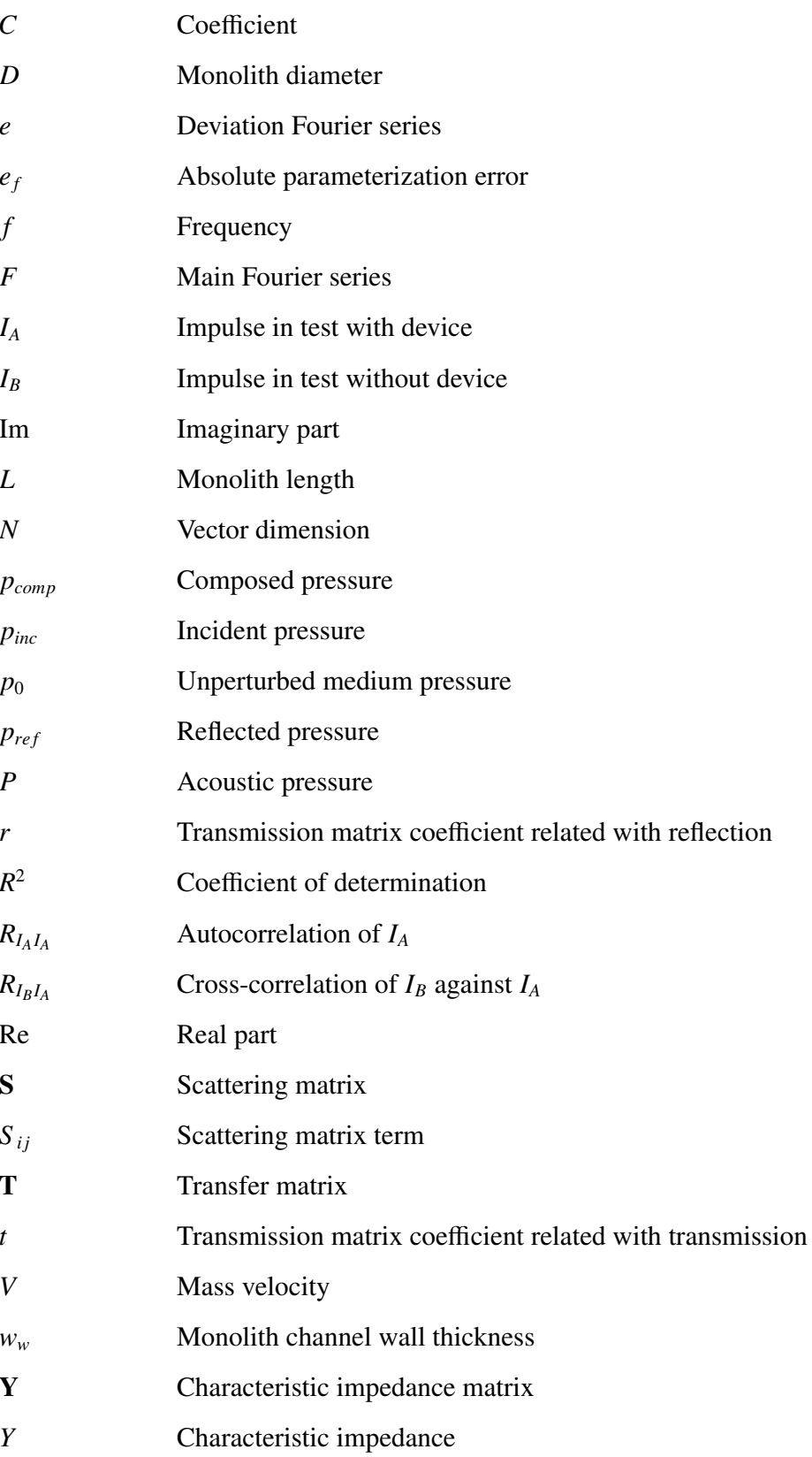

Greek letters

$\Delta \quad$ Transmission matrix determinant 


$\begin{array}{ll}\pi & \text { Characteristic periods ratio } \\ \sigma & \text { Cell density } \\ \tau & \text { Characteristic period of main Fourier series } \\ \tau^{\prime} & \text { Characteristic period of deviation Fourier series }\end{array}$

$\begin{array}{ll}\text { Acronyms } & \\ \text { OD } & \text { zero-dimensional } \\ \text { 1D } & \text { one-dimensional } \\ \text { DOC } & \text { Diesel oxidation catalyst } \\ \text { DPF } & \text { Diesel particulate filter } \\ \text { EATS } & \text { Exhaust aftertreatment system } \\ \text { GPF } & \text { Gasoline particulate filter } \\ \text { LNT } & \text { Lean NOx trap } \\ \text { POC } & \text { Particle oxidation catalyst } \\ \text { SCR } & \text { Selective catalyst reduction } \\ \text { TWC } & \text { Three-way catalyst }\end{array}$

Subscripts

$b \quad$ Baseline operating point

exp Referred to experimental data

in Inlet

$j \quad$ real or imaginary part of $t$ or $r$ coefficient

mod Referred to fluid-dynamic model

$n \quad$ Fourier series order

out Outlet

$p \quad$ Arbitrary operating point

pr Prediction

\section{Introduction}

Noise emission abatement in internal combustion engines is performed by the design of particular mufflers tuned to specific applications and market demands. The ability and design flexibility of these systems for noise attenuation, which is based on both dissipative and reactive features [1], has been widely proved from theoretical and experimental approaches [2]. Nevertheless, the increasingly constraining regulations on pollutant emissions, with US [3] and Europe [4] in front, have led to the inclusion of additional devices in the exhaust line. These exhaust aftertreatment systems (EATS) are primary devoted to gas and aerosol emission abatement [5]. However, there is also general consensus on the fact that through flow [6] and wall-flow [7] monolithic structures act as reactive and dissipative silencers due to its non-negligible influence on the unsteady wave dynamics [8]. 
The presence of EATS produces variations in the flow evolution upstream of the muffler. These comprise diffusion and expansion in the outlet and inlet volumes of each device, as well as local expansion and diffusion at the inlet and outlet interfaces of the monolith, respectively. Similar processes take place between monoliths separated by an intermediate chamber, as in devices composed by a Diesel Oxidation Catalyst (DOC) and a Diesel Particulate Filter (DPF) which are usual in Diesel engines for the control of unburnt hydrocarbons (HC), CO and particulate matter [5]. The removal of nitrogen oxides (NOx) takes usually place in isolated bricks. In heavy-duty Diesel engines, the leading concept for NOx removal is the Selective Catalyst Reduction (SCR) system, which presents higher conversion efficiency than Lean-NOx Trap (LNT) devices, and allows running the engine at maximum efficiency in fuel-sensitive applications [9]. In small Diesel engines, NOx abatement in the aftertreatment is to be performed by LNT devices, which exhibit lower cost than SCR [10] and are likely to substitute [11] or complement [12] the DOC . For these applications, the combination of LNT and SCR presents significant advantages in terms of increase in NOx conversion efficiency and of reduction in ammonia slip [13]. Other solutions being explored are related to the inclusion of NOx-removal functions into the DPF substrate [14] as a way to reduce the cost of the monoliths and the space requirements. Recently, non-ceramic solutions such as the particle oxidation catalyst (POC) are also being considered [15]. In gasoline engines, the Three-Way Catalyst (TWC) is a single device responsible of $\mathrm{CO}, \mathrm{HC}$ and NOx emission reduction. However, new gasoline engine generations are requiring the inclusion of a Gasoline Particulate Filter (GPF) monolith to comply with particulate matter regulations [16].

Besides the canning and layout, the flow evolution is highly affected also by the characteristics of the ceramic monoliths, which generate a non-negligible dissipative effect upstream of the muffler [17] different for through-flow catalyst reactors and wall-flow particulate filters. In the latter, the alternatively plugged ends in channels and the variable properties of the porous substrate play a major role on the acoustic response. In fact, the variations of porous wall permeability and particulate layer thickness as a function of the soot loading determine the magnitude of the dissipative response [18]. In addition, the main features of the reactive response are also dependent on the soot loading and distribution [19].

The amount of different parameters determining the noise reduction capabilities of EATS makes its optimization a complex task. As an additional constraint, reduction targets in pollutant emissions with as low as possible penalty in fuel consumption, i.e. minimizing pressure drop [20], must be kept. Nevertheless, the mere presence of EATS has positive effects on noise damping [21]. Therefore, muffler design must adapt to this boundary and even take advantage for volume and pressure drop reduction [22].

The full exploitation of the potential acoustic advantages of EATS requires the availability of suitable models. The basic physics underlying the acoustic behaviour of the different elements is well understood and suitable onedimensional models have been proposed in the literature, both in the frequency domain $[6,7,17]$ and in the time domain [23]. Despite solutions based on finite element methods [24, 25], the assumption of one-dimensional propagation is fairly reasonable for the small ducts in the monolith bricks [17]. However, this is not the case for the inlet and outlet canning volumes or even for the intermediate gaps between bricks. Therefore, methods including some 
accountancy for three-dimensional wave propagation in those cases have been presented, such as that proposed by Hua et al. [22] in which the model developed in [17] was coupled to a finite element computation, and by Jiang et al. [26], who applied the boundary element method to evaluate the transmission loss of mufflers coupled to catalytic converters or diesel particulate filters. However, the geometrical complexity of many devices (as a result of catalyst light-off requirements that force their installation very close to the engine, with the subsequent packaging constraints) makes still necessary in many cases to rely on experimental measurements. The problem with experimental results is that it is not in general possible to account for all the diversity of flow conditions under which these devices operate.

In this paper, a phenomenological methodology is proposed by which it is possible to account for the influence of the flow conditions on the acoustic response of an aftertreatment device in such a way that experimental results obtained under certain flow conditions may be corrected in order to provide a more accurate picture of the acoustic behaviour of the device in real engine exhaust conditions. Transmission and reflection coefficients of different aftertreatment devices were measured under different excitation amplitudes and superimposed mean flows, and the observed influence was parameterized in a simple and efficient way. It was also found that extrapolation of the results to other flow conditions was possible making use of information obtained from a gas dynamic model. Results are accurate despite these models present high dependence on the numerical method and mesh definition to provide an accurate computation in the frequency domain [27], and on the time-marching approach between the different 1D, 0D and quasi-steady elements defining the EATS geometry [28]. The limitations coming from the simplification of the actual geometry, which play an important role in the frequency domain modelling, were also overcome. It is due to the fact that the calculation in the time domain is very sensitive to the propagation velocity and this feature is also retained in the frequency domain regardless of the ability to capture the acoustic response.

The paper is organized as follows. First, the methodology is described in detail, comprising: the measurement set-up and the associated signal processing, the two-step Fourier series approach used for the parameterization of the acoustic response obtained, and the use of gas-dynamic modelling to incorporate the convective effects. Once the methodology is described, it is applied to the evaluation of the transfer matrices and transmission loss of different EATS, showing the potential of the methodology. Finally, the main conclusion of the work are outlined.

\section{Phenomenological methodology}

As commented above, the procedure proposed to account for the effect of flow conditions on the acoustic response of an EATS device results from a combined experimental and computational approach. The steps of the methodology are sketched in Figure 1. The different steps are described in detail in the following paragraphs.

\subsection{Experimental characterization}

According to the flow-chart shown in Figure 1, the first step of the proposed methodology is devoted to the experimental characterization of the device acoustic response. It is performed in an arbitrary operating point, which 
is used as baseline. Tests were conducted at room temperature in an impulse test rig [29]. This rig allows exciting the tested device with a pressure pulse of controlled amplitude and duration in order to account for the influence of the flow conditions. These tests can be performed with or without mean flow to analyze the influence of convective effects separately. Figure 2 shows a scheme of the installation and its operating principle.

The purpose of this rig is to provide suitable conditions to assess the reflection and transmission characteristics of the device, so that any influence of reflections from the pipe ends of the gas stand is avoided. The incident pulse is generated at room temperature by means of a high-speed electrovalve that controls the discharge from a pressurized air tank. The amplitude of the pressure pulse is governed by the pressure tank and the electrovalve opening duration, which in turn sets the pulse duration.

The electrovalve is connected to a long duct through which the incident pulse propagates. The length of the propagation duct up to the EATS is selected to ensure that the pressure transducer 1 only measures the generated pressure pulse once it is completely developed. As sketched in Figure 2, there is no overlap with the incoming pulse reflected by the device.

When the incident pulse arrives at the EATS it generates a reflected wave that travels back to the electrovalve, which is kept closed. The superposition of the incident and reflected pulses $\left(p_{\text {comp }}\right)$ is registered by pressure transducer 2. Finally, pressure transducer 3 registers the pressure pulse transmitted downstream of the EATS. A long propagation duct is also placed at the device outlet. Thus, the measurement of the transmitted pressure is carried out without any influence of the reflection coming back from the discharge end.

As described, pressure transducer 2 registers the superposition of the incident and reflected pulses at the device inlet. To characterize the acoustic response it is necessary to determine the isolated reflected wave. To do that an additional test with a straight duct portion instead of the EATS is performed. Firstly the EATS is tested being subjected to several incident pressure pulses of similar duration and amplitude (repeatability within a prescribed uncertainty). Next the EATS is replaced by the straight duct to be tested. Again, it is subjected to several measurements with incident pressure pulses similar to those exciting the EATS. The post-processing consists of comparing the measurements of the generated pulse, i.e. the pressure recorded by transducer 1, in order to find the most coincident pair of EATS and duct tests by applying a statistical procedure. This is done by evaluating the difference between the autocorrelation of the pulse recorded by transducer 1 in the test without device (impulse $\mathrm{B}, I_{B}$ ), and the cross correlation between the pulses recorded with (impulse A, $I_{A}$ ) and without device [30]. In [29] it is indicated that the repeatability of the pulse generator provides values of this difference below $5 \%$, which is considered to be sufficient in practice. In particular, in order to evaluate the effect of a given generated pulse on the tested device, a sample of tests consisting of 5 tests with device and 10 tests without the device (straight duct) is considered. Then, the method is applied to find the pair of tests fulfilling, in the limit,

$$
\left|\frac{R_{I_{B i} I_{A i}}}{R_{I_{A i} I_{A i}}}\right|=1
$$


where $R_{I_{B i} I_{A i}}$ is the cross-correlation of $I_{B i}$ with respect to $I_{A i}$ and $R_{A i A i}$ is the autocorrelation of $I_{A i}$. According to this procedure, the dispersion of the selected pair of tests is limited to be not higher than $5 \%$, i.e.

$$
\left|1-\frac{R_{I_{B i} I_{A i}}}{R_{I_{A i} I_{A i}}}\right| \leq 0.05
$$

Once the tests are selected, the incident pressure pulse $\left(p_{\text {inc }}\right)$ at the EATS inlet is assumed to be that measured by the pressure transducer 2 in the straight duct portion test. Known the EATS incident pressure and making use of the measurement of the pressure transducer 2 in the EATS test is possible to calculate the reflected pressure pulse $\left(p_{\text {ref }}\right)$ by applying Eq. $3[31,32]$

$$
\left(\frac{p_{r e f}}{p_{0}}\right)^{\frac{\gamma-1}{2 \gamma}}=\left(\frac{p_{\text {comp }}}{p_{0}}\right)^{\frac{\gamma-1}{2 \gamma}}-\left(\frac{p_{\text {inc }}}{p_{0}}\right)^{\frac{\gamma-1}{2 \gamma}}+1,
$$

where $p_{0}$ is the pressure of the unperturbed medium.

This process is repeated twice in order to obtain the transmission matrix coefficients of every EATS. Firstly, the direct test is performed. In this test the device is excited with an incident pressure pulse travelling from the inlet to the outlet. In this way, the reflection at the inlet interface and the transmission from inlet to outlet can be assessed. The inverse test is next performed with the same procedure, but in this case the device is excited from the outlet to the inlet, so that the reflection at the outlet interface and the transmission from outlet to inlet can also be determined. As an example of the raw results obtained, in Figure 3 the incident, transmitted and reflected pressure pulses measured in the direct and inverse tests of the device composed by DOC-1 and DPF-1 are shown. The main geometric data of these devices are detailed in Table 1, which also includes the characteristics of each EATS used. The baseline operating point was defined by an amplitude and duration of the generated pulse of 150 mbar and 14 ms respectively and without any superimposed mean flow. This pulse was tested in all the devices. Additionally, other operating points considering different pressure pulses and mean mass flows across the device were tested in order to account for the influence of convective transport. The pulse profiles provided by the impulse test rig are representative of the pressure pulses impinging on the elements placed in the engine tailpipe when it operates in realistic conditions. It is discussed by Broatch et al. [29], who evaluated the modified impulse method used in this work showing its ability to allow for the study of the frequency response of acoustic filters when subject to weakly non-linear pulses. Nonlinear issues in pulse propagation and interaction were addressed with the proposed method in an approximate but sufficiently accurate fashion, as verified by means of numerical unsteady flow calculations.

According to the basis of the impulse method [29], which was validated against one-dimensional unsteady nonlinear flow calculations, the pressure pulse is propagated non-linearly along the inlet duct. Therefore the non-linear development of the pressure pulse leads to the formation of an asymptotic singularity. However, once the pulse has reached the asymptotic region any further non-linear development should be scarcely noticeable, both in the time and the frequency domains. This occurs somewhere upstream of transducer 2, as evidenced in Figure 3, where incident pressure pulses are represented. 
As a result of the procedure just described, by transforming the measurement results into the frequency domain, all the transmission matrix coefficients can be obtained as a function of frequency, and thus one may write

$$
\left[\begin{array}{c}
P_{\text {in }}^{-} \\
P_{\text {out }}^{+}
\end{array}\right]=\left[\begin{array}{ll}
r_{1} & t_{12} \\
t_{21} & r_{2}
\end{array}\right]\left[\begin{array}{c}
P_{\text {in }}^{+} \\
P_{\text {out }}^{-}
\end{array}\right]
$$

where $P_{\text {in }}^{+}$and $P_{\text {out }}^{-}$represent the incident sound pressure spectra at the inlet and the outlet, respectively, whilst $P_{\text {in }}^{-}$ and $P_{\text {out }}^{+}$refer to the sound pressure spectra resulting from the device acoustic response, i.e. from its reflection and transmission properties.

Once the transmission matrix coefficients for a particular excitation are obtained, the transfer matrix can be computed from the scattering matrix and from the characteristic impedances at the inlet and the outlet interfaces of the device, in particular where transducer 2 and 3 are located. The well-known frequency domain relation between wave components and acoustic pressure $P$ and mass velocity $V$ can be written in compact matrix form as

$$
\left.\begin{array}{l}
P=P^{+}+P^{-} \\
V=\frac{1}{Y}\left(P^{+}-P^{-}\right)
\end{array}\right\} \Rightarrow\left[\begin{array}{l}
P \\
V
\end{array}\right]=\underbrace{\left[\begin{array}{cc}
1 & 1 \\
Y^{-1} & -Y^{-1}
\end{array}\right]}_{\mathbf{Y}}\left[\begin{array}{c}
P^{+} \\
P^{-}
\end{array}\right]
$$

Considering that any influence of the mean flow or the pulse amplitude on the characteristic impedance $Y$ would arise as a complex correction term associated with friction losses [1], which becomes important only at Mach numbers much higher than those considered in this work (see below), it was assumed that it would suffice to consider the no flow expression of Y, namely

$$
Y=\frac{a}{A}
$$

where $a$ is the speed of sound and $A$ represents the cross-section area, consistently with the choice, as variables, of pressure and mass velocity fluctuations. Now, the scattering matrix relates forward and backward wave component spectra at the inlet and the outlet of the device, as

$$
\left[\begin{array}{c}
P_{\text {in }}^{+} \\
P_{\text {in }}^{-}
\end{array}\right]=\left[\begin{array}{cc}
S_{11}=\left(t_{12}\right)^{-1} & S_{12}=-r_{2}\left(t_{12}\right)^{-1} \\
S_{21}=r_{1}\left(t_{12}\right)^{-1} & S_{22}=-\Delta\left(t_{12}\right)^{-1}
\end{array}\right]\left[\begin{array}{c}
P_{\text {out }}^{+} \\
P_{\text {out }}^{-}
\end{array}\right],
$$

where $\Delta$ is the determinant of the transmission matrix as defined by Eq. 3. Then, by combining Eqs. 5 and 7 the transfer matrix relating pressure and mass velocity fluctuations can be finally written as:

$$
\underbrace{\left[\begin{array}{ll}
T_{11} & T_{12} \\
T_{21} & T_{22}
\end{array}\right]}_{\mathbf{T}}=\underbrace{\left[\begin{array}{cc}
1 & 1 \\
Y_{\text {in }}^{-1} & -Y_{\text {in }}^{-1}
\end{array}\right]}_{\mathbf{Y}_{\text {in }}} \underbrace{\left[\begin{array}{cc}
S_{11} & S_{12} \\
S_{21} & S_{22}
\end{array}\right]}_{\mathbf{S}} \underbrace{\frac{1}{2}\left[\begin{array}{cc}
1 & Y_{\text {out }} \\
1 & -Y_{\text {out }}
\end{array}\right]}_{\mathbf{Y}_{\text {out }}^{-1}}
$$



i.e.

\subsection{Coefficient parameterization}

The use of experimental data to characterize the acoustic response of the devices presents three main limitations. Firstly, experimental data are a discrete function of the frequency. Additionally, data storage requirements increase with the operation range covered but also with the sampling frequency and the frequency range of interest. Finally, measurement uncertainties can arise related to transducer characteristics and also to the need to combine the results of direct and inverse tests to compute the transfer matrix.

In order to overcome these limitations, every real and imaginary part of the transmission matrix coefficients was parameterized by means of a Fourier series, this being suggested by explicit solutions for simple basic systems such as ducts and by the quasi-periodic and sinusoidal-like profile observed in the transmission matrix coefficients. Therefore, the real or imaginary parts of all the transmission matrix coefficients were expressed as

$$
F_{b, j}(f)=a_{0, j}+\sum_{k=1}^{n}\left(a_{k, j} \cos \left(k \tau_{b, j, \exp } f\right)+b_{k, j} \sin \left(k \tau_{b, j, \exp } f\right)\right),
$$

where $F_{b, j}(f)$ represents the parameterized real or imaginary part of the transmission matrix coefficients (indexed by subscript $j$ ) obtained experimentally for the baseline operating point $(b) ; a_{k, j}$ and $b_{k, j}$ are the Fourier series coefficients; and $\tau_{b, j, \text { exp }}$ is the characteristic period in the Fourier series for the baseline operating point.

Figure 4 shows the resulting parameterization of the transmission matrix coefficients (real and imaginary parts) of DOC+DPF-2 in the baseline direct test. The parameterization is directly applied to the real and imaginary parts of every coefficient fitting the experimental values to an 8th order Fourier series by applying the trust region algorithm [35] in order to minimize the error computed by non-linear least squares [36]. The modulus of the transmission matrix coefficients are represented in plots (c) and (f) in Figure 4. Modulus parameterization is also very accurate although with a slightly lower coefficient of determination because of the fitting error propagation.

Despite the good results shown in Figure 4, the application of the described process to the whole population of tests provided significant deviations in the modulus of the coefficients for particular cases. An example corresponding to the baseline operating point in the direct test of SCR-1 is shown in Figure 5. Series in red colour, which is labelled as one-step fitting, has been obtained by fitting the experimental data to Eq. 9. It is observed that the low value of the coefficient of determination in both the real and imaginary parts of the coefficients propagates dramatically to the modulus. This is actually over-smoothed, resulting in the complete loss of the reactive response in the parameterized coefficient modulus.

Due to the occurrence of this kind of results, the described procedure was improved by parameterizing the deviation between the experimental data and the first Fourier series. This deviation is again fitted to a Fourier series,

$$
e_{b, j}(f)=a_{0, j}^{\prime}+\sum_{k=1}^{n}\left(a_{k, j}^{\prime} \cos \left(k \tau_{b, j, e x p}^{\prime} f\right)+b_{k, j}^{\prime} \sin \left(k \tau_{b, j, e x p}^{\prime} f\right)\right)
$$


so that the whole procedure constitutes a two-step Fourier series parameterization, the experimental data being represented by the addition of $F_{b, j}(f)$ and $e_{b, j}(f)$. The second Fourier series (deviation function) is obtained by applying the same procedure as in the first-step.

The results obtained from the application of the two-step Fourier series parameterization are plotted in Figure 5 in blue colour. It is clearly observed how the fitting of both the real and imaginary parts of the coefficients is greatly improved leading to an accurate prediction of the modulus.

The great advantage of this Fourier-series-based procedure is the robustness it shows to reproduce the shift that transmission matrix coefficients exhibit when the propagation velocity varies. This is of course the case when the mean flow is changed, as this directly affects the convective transport, but also when the amplitude of the incident wave changes, as a change in the amplitude produces the variation of the maximum propagation velocity. According to the definition of the Fourier series, the shift is mainly governed by the characteristic period whilst the coefficients $a_{k, j}$ and $b_{k, j}$ control the amplitude of the function and are specific to each particular device, i.e. are representative of the geometry. This ability of the Fourier series is shown in Figures 6 and 7. In these figures the fittings of additional operating points different from the baseline and corresponding to DOC+DPF-1 are represented. In particular, Figure 6 shows the effect of a change in the amplitude of the pressure pulse in comparison with the baseline condition; Figure 7 depicts the case of considering a superimposed mean flow. In addition to the particular operating point, each plot includes in black colour the experimental data corresponding to the baseline operating point. Plots in Figure 6 make evident that the variation of the excitation amplitude shifts the reflection coefficient $\left(r_{2}\right)$. The transmission coefficient $\left(t_{21}\right)$ is also shifted but in lower magnitude than in the case of reflection. Nevertheless, the superimposition of a mean flow leads to negligible effects on the reflection coefficient due to the compensation of the convection transport caused by the mean flow travelling in the same direction as the reflected wave. In this case, the change in the transmission coefficient becomes relevant due to the superposition of the mean flow convection from the inlet to the outlet on the transmitted wave travelling from the outlet to the inlet in the inverse test.

In order to fit the experimental data when the baseline conditions change, the Fourier series coefficients obtained for the baseline operating point were kept constant both for the first and the second steps. The characteristic period of the deviation function (second-step) was also imposed to be the same as that obtained in the fitting of the baseline operating point for the real and imaginary parts of each transmission matrix coefficient. Therefore, only the characteristic period of the main Fourier series (first-step) was modified to capture the shift suffered by the coefficients. The dependence on just one parameter, i.e. the characteristic period of the main Fourier series, is a key result to verify the potential of the proposed methodology in this work as a tool useful for prediction, what is discussed in following sections. Notice that the deviation function could not be varied for prediction purposes since it is unknown by definition. As shown in Figure 8, which presents the decomposition of the two-step fitting corresponding to the case shown in Figure 5, the value of the deviation function is small and provides only additional information allowing to improve the accuracy of the main (first) Fourier series, which is the one containing the most relevant information related to the acoustic response of the device. It is defined for each operating point as 


$$
F_{p, j}(f)=a_{0, j}+\sum_{k=1}^{n}\left(a_{k, j} \cos \left(k \tau_{p, j, \exp } f\right)+b_{k} \sin \left(k \tau_{p, j, \exp } f\right)\right),
$$

where $p$ refers to an excitation different than that of the baseline and $\tau_{p, j, \text { exp }}$ is the characteristic period for the real and imaginary parts of the transmission matrix coefficients ( $j$ subscript). Its value is determined by minimizing the residuals of the fitting process applying the Nelder-Mead simplex method [37]. These are defined as the root mean square value of the absolute deviation between the experimental coefficients $\left(C_{p, j, \exp }\right)$ and the two-step Fourier series parameterization evaluated at each discrete frequency for which experimental data are available:

$$
\begin{gathered}
\tau_{p, j, \exp } / \min (S)=\min \left(\frac{1}{N} \sqrt{\sum_{f}^{N} e_{f}^{2}\left(\tau_{p, j, \exp }\right)}\right) \\
e_{f}\left(\tau_{p, j, \exp }\right)=C_{p, j, \exp }(f)-F_{p, j}\left(f, \tau_{p, j, \exp }\right)-e_{b, j}(f)
\end{gathered}
$$

Figure 9 shows the impact of a $\pm 5 \%$ change of the best characteristic period on the difference between the experimental data and the two-step Fourier series fitting. Despite the small variations considered in the characteristic period, $S$ experiments a sharp increase.

At this point it is interesting to analyse some additional parameters that might affect the accuracy and robustness of the proposed methodology. On one hand, the frequency step of the experimental data is $4 \mathrm{~Hz}$. This value is the minimum one possible since it is imposed by the measurement window used in the impulse test rig, as this frequency step is precisely the inverse of the measurement window $(0.25 \mathrm{~s})$, whose maximum size is limited to allow for the isolation of the measured pulses from the pulses reflected at the open pipes ends. Any reduction of the frequency step while ensuring the correct isolation of the pulses would require the use of longer ducts what would cause operative problems. Figure 10 shows that a change from $4 \mathrm{~Hz}$ to $16 \mathrm{~Hz}$ in the frequency step of the experimental data does not affect significantly the accuracy of the results provided by the proposed methodology because of its inherent smoothing effect.

Another relevant parameter is the maximum frequency considered in the fitting process. The value considered here $(2000 \mathrm{~Hz})$ clearly exceeds the usual range analysed in an ICE exhaust. For instance, the maximum frequency of interest in an in-line four-cylinder engines is usually taken to be that corresponding to the fourth multiple of the firing frequency at maximum engine speed, that is, $800 \mathrm{~Hz}$ at $6000 \mathrm{rpm}$. Anyway, the proposed methodology is able to deal with different maximum frequencies without any significant impact on the accuracy. This can be checked in Figure 11, where results obtained setting the maximum frequency to $1000 \mathrm{~Hz}$ and $3000 \mathrm{~Hz}$ are shown, and differences in the accuracy of the fitting are not relevant. However, further increments of the maximum frequency would probably require an increase in the Fourier series order. 


\subsection{Fluid-dynamic modelling}

The application of gas dynamics models to the prediction of the acoustic response in internal combustion engine exhaust systems is a common practice. Nevertheless, the accuracy of the frequency domain results is very dependent on diverse parameters. Several works address this topic focusing on the influence of the way in which the time-step is fixed in the different model elements [28] and on the impact of the numerical method and the mesh size [33]. Different model approaches have also a relevant influence on the frequency domain results. In fact, small differences in the time domain can lead to more apparent deviations in the frequency domain [34]. In this way, experimental and modelled results in the time domain are compared in Figure 12. GT-Power software was applied to model the time domain response of TWC-1 against excitations of different amplitude in direct and inverse tests. In addition, two model setups, whose only difference was a slight change in the length of inlet and outlet pipes, were considered. The inlet duct length was increased whereas the outlet one was shortened in setup 2. In direct tests, these length variations hardly affected the transmission, but the reflection was clearly delayed. The transmission in the inverse tests showed more sensitivity to this geometry change resulting in a noticeable delay due to the length increase of the inlet duct. In addition, the maximum magnitude of the transmitted pulses was better predicted but at the expense of a shorter wave duration. Concerning the reflected wave, shifts appeared when the pipe length was varied both in direct and inverse tests. As expected, the sign of the shift is opposite between direct and inverse tests, due to the respective increase or decrease in the length.

Despite the influence produced by inlet and outlet length variations is small in the time domain, the response in the frequency domain shows to be very sensitive. A summary of the results in the frequency domain is represented in Figure 13, which shows the real part of the transmission matrix coefficients for each operating point. For the sake of clarity, because of the high number of series, plots (a) and (b) are referred to experimental data whereas plots (c) and (d) include the modelled results for each model setup. As previously shown in Figure 6 devoted to DOC+DPF-1, plots (a) and (b) in Figure 13 evidence that the change in the amplitude of the excitation, i.e. in the characteristic propagation velocity, produces the shift of the transmission matrix coefficients. Again, a change only in the amplitude mainly affects the reflection coefficient, both in the direct and inverse cases. The shifts in $t_{12}$ and $t_{21}$ are small and noticeable only at high frequencies (above $1000 \mathrm{~Hz}$ ). These shifts are also observed in plots (c) and (d) for the modelled transmission matrix coefficients regardless of the model setup. Nevertheless, the accuracy of the gas dynamic modelling in the frequency domain is very dependent on the specific setup. Results from setup 1 captured the TWC-1 response, especially in the case of the transmission. Some deviations appeared in the reflection coefficient covering the band between 1100 and $1500 \mathrm{~Hz}$ in the direct test and in the frequency range above $1600 \mathrm{~Hz}$ in the inverse test. In contrast to setup 1, results from setup 2 were not able to provide the right trend of the acoustic response of the TWC-1, not even in the low frequency range.

Beyond the degree of accuracy of the model in the frequency domain, the ability to predict the shift in the transmission matrix coefficients due to a change in the characteristic propagation velocity between operating points is an outcome from gas dynamic modelling that can be exploited. Based on this feature, the ratio of the characteristic peri- 
ods defining the Fourier series for every component (real and imaginary parts) of the transmission matrix coefficients between the baseline and any other operating point must coincide when comparing experimental and modelled data:

$$
\pi_{p, j}=\frac{\tau_{p, j, \text { exp }}}{\tau_{b, j, \text { exp }}}=\frac{\tau_{p, j, \text { mod }}}{\tau_{b, j, \text { mod }}}
$$

Therefore, the ratio $\pi_{p, j}$ is representative of the device response in operating point $p$ once a baseline condition has been experimentally characterized. The characteristic period used is that of the main Fourier series. The reason is that it is the one manifesting the sensitivity to the change in the propagation velocity as previously discussed in Figures 6 and 7. As sketched in Figure 1 and based on Eq. 14, once the ratio $\pi_{p, j}$ is known from modelling results, it is then possible to predict the characteristic period at any operating point. For this purpose $\pi_{p, j}$ must be combined with the characteristic period coming from the parameterization of the experimental baseline operating point:

$$
\tau_{p, j, p r}=\pi_{p, j} \tau_{b, j, e x p}
$$

In summary, the application of the two-step Fourier series parameterization of the experimental baseline transmission matrix coefficients provides a set of constants defining the specific acoustic response of the EATS. In parallel, simple gas dynamic modelling, which can be even inaccurate in the time and the frequency domains, is able to provide a good prediction of the characteristic period provided that this is known from experiments for the baseline (Eq. 15). Therefore, all the information required to extrapolate the experimental transmission matrix coefficients is available.

As an example, Figure 14 shows the prediction of the coefficients obtained with the described methodology. They correspond to the direct test of TWC-1 excited by the pressure pulse of 230 mbar. The methodology was applied to the results from setups 1 and 2 of the gas dynamics model. The agreement with experimental data is remarkable for both setups, despite the disagreements in the gas dynamic modelling. Deviations in the real and imaginary parts are very small. Consequently, the shape of the modulus, which is shown in Figure 14(c) and (f), is also predicted with great accuracy.

The potential of the described methodology is also shown in Figure 15, in which the DOC+DPF-2 response is considered. In this example, the gas dynamic model (red series) is not able to reproduce the acoustic response. However, the model ability to account for the difference between the baseline and different operating points made possible to predict the transmission matrix coefficients applying the phenomenological methodology. Results from its application are plotted in blue colour. As in TWC-1, the real and imaginary parts of the coefficients are reproduced with precision resulting in a proper reproduction of the modulus. In fact, the accuracy is of the same order of magnitude as that of the best Fourier series fitting, as it can be checked against the set of results from fitting shown in Section 2.2.

\section{Transfer matrix and transmission loss prediction}

As a final step, the transfer matrix and the transmission loss are computed applying the extrapolated transmission matrix. The transfer matrix coefficients of TWC-1 corresponding to the excitation with a pressure pulse of $230 \mathrm{mbar}$ of 
amplitude are shown in Figure 16. The result of the described phenomenological methodology, which is represented by the blue series, is compared to experimental data and to the direct fitting of these data by means of Fourier series. The results evidence similarities between the prediction and the direct fitting of the experimental data obtained by minimizing the error of the two-step Fourier series, as already observed in transmission matrix coefficients. The results obtained are very accurate, with only some deviations appearing mainly around spurious spikes in the experimental data and coming from the measurement process. In Figure 17 an example of transmission loss prediction without mean flow in SCR-1 is given. As in the case of the transfer matrix prediction, this magnitude also shows good agreement between experimental and predicted data in the whole frequency range. These results completely confirm both the ability of the gas dynamic modelling to account for the change in the characteristic period, and that the Fourier series coefficients obtained for the baseline conditions are particular to each device.

The overshoots are due to the limitations of the experimental procedure. In fact, the determination of the transfer matrix requires the combination of four tests, i.e. device and straight duct for direct and inverse excitations. Therefore, small mismatches in the reproduction of the excitations between inverse and direct tests as well as variations in room conditions may result in transfer matrix disturbances at certain frequencies. A solution to remove the non-physical content of the transfer matrix without loss of acoustic information is the application of the two-step Fourier series parameterization, provided that the modulus of the transmission matrix coefficients is properly reproduced, as shown in Figure 5. Cases with mean flow are especially sensitive to overshoots in experimental data. Figure 18 represents the transfer matrix in TWC-1 for the case of $100 \mathrm{~kg} / \mathrm{h}$ in mean mass flow. This corresponds to Mach 0.048 , which is evaluated at the inlet pipe of the set-up, which in turn has the same diameter as the inlet flange of the device. In spite of the fact that the transfer matrix computation propagates the errors present in the transmission matrix coefficients, the prediction of the characteristic period in the first Fourier series provides suitably smoothed transfer matrix coefficients while avoiding non-physical spikes. This feature is present in both the real and imaginary parts of all the coefficients. Again, the solution is very close to the experimental data but with the advantages of smoothing, and showing an accuracy of the same order of magnitude as that of the best two-step Fourier series fitting. As represented in Figure 19, the direct and inverse transmission losses are also correctly predicted under mean mass flow operating conditions, thus confirming the robustness of the proposed methodology to predict the different magnitudes describing the acoustic response.

\section{Summary and conclusions}

A phenomenological methodology allowing the correction of the experimental acoustic response of exhaust aftertreatment devices for the effect of pressure perturbation characteristics and flow conditions has been presented. Even though the work was focused on these particular systems, the procedure might be potentially applied to other systems in which a linear shift of the transmission matrix coefficients with the flow velocity may be identified. However, devices exhibiting internal flow features with a non-linear dependence on flow velocity, as for instance in the 
case of the flow separation and the end correction at the entrance of a side-branch resonator, should be investigated in particular studies.

The proposed methodology combines experimental and modelling tools to assess changes in transfer matrix due to variations of the characteristic propagation velocity of the pressure waves. The first step consists of the experimental determination of the acoustic response of the EATS against an arbitrary excitation. This serves as a baseline to predict the change induced by the operating conditions in the transfer matrix or, in particular, in the transmission matrix coefficients.

Secondly, the experimental transmission matrix coefficients were parameterized. A two-step Fourier series parameterization was proposed to avoid the loss of relevant physical information in the correlated functions, specially in what is related to the reactive response of the device. It has been shown how the constants of both Fourier series and the characteristic period of the second one, which fits the deviation coming from the main series, are representative of a given device, i.e. of its geometry. Therefore, any change produced by the operating conditions can be related to variations in the characteristic period of the main Fourier series. The potential of this property was explored by parameterizing gas dynamic modelling results.

Then, the final step was focused on the predictive capability assessment based on simple gas dynamic modelling of the device. The ratio between the characteristic period predicted by the model for the main Fourier series in two different operating points (one of them being the baseline) is also valid for the experimental (actual) response. This ratio has been shown to be correct even when the gas dynamic modelling is not accurate neither in the time nor in the frequency domains, so that it is not able to predict properly the absolute value of the characteristic period.

Finally, once such ratio, which represents the influence of the operating point, is known, by combining it with the characteristic period coming from the parameterization of the experimental baseline condition it is possible to estimate the characteristic period of the main Fourier series at any operating condition. The predicted value for this parameter together with the set of constants defining the specific acoustic response of the EATS obtained from the baseline experiments provide all the information required to compute the transmission matrix coefficients. Consequently, the transfer matrix is uniquely defined. Good accuracy in the results obtained for the transmission matrix, the transfer matrix and the transmission loss has been obtained for different EATS covering variations in pressure pulse amplitude and duration, as well as in mean flow.

\section{Acknowledgments}

This work has been partially supported by the Spanish Ministerio de Economía y Competitividad through Grant No. TRA2013-40853-R. Additionally, the Ph.D. student E.J. Sanchis has been funded by a grant from Universitat Politècnica de València with reference FPI-2016-S2-1355. These supports are gratefully acknowledged by the authors. Finally, the authors wish also to acknowledge the suggestions of the referees, which have resulted in a considerable improvement of the text. 
[1] M. Munjal, Acoustics of ducts and mufflers, John Wiley \&Sons, New York, 1987.

[2] B.S. Sridhara, M.J. Crocker, Review of theoretical and experimental aspects of acoustical modeling of engine exhaust systems, Journal of the Acoustical Society of America 95 (5) (1994) 2363-2370.

[3] Control of air pollution from motor vehicles: Tier 3 motor vehicle emission and fuel standards, Proposed Rules, Federal Register, vol. 78, No 98 (May 2013)

[4] Regulation (EC) no 715/2007 of the European Parliament and of the Council of 20 June 2007 on type approval of motor vehicles with respect to emissions from light passenger and commercial vehicles (Euro 5 and Euro 6) and on access to vehicle repair and maintenance information, Official Journal of the European Union (June 2007).

[5] T.V. Johnson, Review of vehicular emissions trends, SAE Technical Paper 2015-01-0993, 2015.

[6] A. Selamet, V. Easwaran, J.M. Novak, R.A. Kach, Wave attenuation in catalytic converters: reactive versus dissipative effects, Journal of the Acoustical Society of America 103 (2) (1998) 935-943.

[7] S. Allam, M. Åbom, Acoustic modelling and testing of diesel particulate filters, Journal of Sound and Vibration 288 (2005) $255-273$.

[8] J.M. Desantes, A.J. Torregrosa, A. Broatch, H. Climent, Silencing capabilities of non-silencer elements: an underused potential?, 4th Styrian Noise, Vibration \& Harshness Congress, Graz, Austria, November 15-17, 2006.

[9] T.V. Johnson, Diesel engine emissions and their control. An overview, Platinum Metals Review 52 (1) (2008) $23-27$.

[10] F. Posada, F. Bandivadekar, J. German, Estimated cost of emission control technologies for light-duty vehicles. Part 2 - Diesel, SAE Technical Paper 2013-01-0539, 2013.

[11] G. Neely, J. Sarlashkar, D. Mehta, Diesel cold-start emission control strategy for 2015-2025 LEV III emissions, SAE International Journal of Engines 6(2) (2013) 1009-1020.

[12] H. Nanjundaswamy, D. Tomazic, C. Severin, A. Kolbeck, T. Körfer, T. Wittka, T. Schnorbus, Further improvement of conventional diesel NOx aftertreatment concepts as pathway for SULEV, DEER Conference, Detroit, US, 2011.

[13] C. Seo, H. Kim, B. Choi, M.T. Lim, C. Lee, De-NOx characteristics of a combined system of LNT and SCR catalysts according to hydrothermal aging and sulfur poisoning, Catalysis Today 164 (2011) 507-514.

[14] W. Kang, B. Choi, Effect of copper precursor on simultaneous removal of PM and NOx of a 2-way SCR/CDPF, Chemical Engineering Science 141 (2016) 175-183.

[15] A. Hynninen, M. Åbom, Simulation of the particle oxidation catalyst POC acoustics, Noise Control Engineering Journal 62 (5) (2014) 368-374.

[16] A. Mamakos, N. Steininger, G. Martini, P. Dilara, Y. Drossinos, Cost effectiveness of particulate filter installation on direct injection gasoline vehicles, Atmospheric Environment 77 (2013) 16-23.

[17] S. Allam, M. Åbom, Sound propagation in an array of narrow porous channels with application to diesel particulate filters, Journal of Sound and Vibration 291 (2006) 882-901.

[18] J. Hicks, W. Hill, A. Kotrba, DPF acoustic performance: an evaluation of various substrate materials and soot conditions, SAE Technical Paper 2011-01-2198, 2011.

[19] A.J. Torregrosa, J.R. Serrano, P. Piqueras, P., O. García-Afonso, Experimental and computational approach to the transient behaviour of wall-flow diesel particulate filters, Energy, In press, http://dx.doi.org/10.1016/j.energy.2016.11.051, 2016.

[20] J.M. Lújan, V. Bermúdez, P. Piqueras, O. García-Afonso, Experimental assessment of pre-turbo aftertreatment configurations in a single stage turbocharged Diesel engine. Part 1: Steady-state operation, Energy 80 (2015) 599-613.

[21] E. Dokumaci, Sound transmission in narrow pipes with superimposed uniform mean flow and acoustic modelling of automobile catalytic converters, Journal of Sound and Vibration 182(5) (1995) 79917808.

[22] X. Hua, D.W. Herrin, T.W. Wu, T. Elnady, Simulation of diesel particulate filters in large exhaust systems, Applied Acoustics 74 (2013) $1326-1332$

[23] G. Montenegro, A. Onorati, A. Della Torre, A.J. Torregrosa, The 3D cell approach for the acoustic modeling of after-treatment devices, SAE International Journal of Engines 4 (2) (2011) 2519-2530. 
[24] K. Peat, A first approximation to the effects of mean flow on sound propagation in capillary tubes, Journal of Sound and Vibration 175 (1994) 47517489.

[25] R.J. Astley, A. Cummings, Wave propagation in catalytic converters: formulation of the problem and finite element solution scheme, Journal of Sound and Vibration 188(5) (1995) 63517657.

[26] C. Jiang, T.W. Wu, M.B. Xu, C.Y.R. Cheng, BEM modeling of mufflers with diesel particulate filters and catalytic converters, Noise Control Engineering Journal 58(3) (2010) 243-250.

[27] J.R. Serrano, F.J. Arnau, P. Piqueras, O. García-Afonso, Application of the two-step Lax\&Wendroff-FCT and the CE-SE method to flow transport in wall-flow monoliths, International Journal of Computer Mathematics 91(1) (2014) 71-84.

[28] J. Galindo, J.R. Serrano, F.J. Arnau, P. Piqueras, High-frequency response of a calculation methodology for gas dynamics based on Independent Time Discretisation, Mathematical and Computer Modelling 50 (5-6) (2009) 812-822.

[29] F. Payri, J.M. Desantes, A. Broatch, Modified impulse method for the measurement of the frequency response of acoustic filters to weakly nonlinear transient excitations, Journal of the Acoustical Society of America 107(2) (2000) 731-738.

[30] A. Broatch, Medida de la respuesta acústica de silenciadores (Measurement of silencers acoustic response), SPUPV-95.2096 (in Spanish), ISBN 84-7721-346-1, Valencia, Spain, 1995.

[31] F.K. Bannister, G.F. Mucklow, Wave action following sudden release of compressed gas from a cylinder, Proceedings of the Institution of Mechanical Engineers 159 (1948) 269-287.

[32] F. Payri, J.M. Desantes, A.J. Torregrosa, Acoustic boundary condition for unsteady one-dimensional flow calculations, Journal of Sound and Vibration 188(1) (1995) 85-110.

[33] A. Broatch, J.R. Serrano, F.J. Arnau, D. Moya, Time-domain computation of muffler frequency response: Comparison of different numerical methods, Journal of Sound and Vibration 305 (2007) 333-347.

[34] A.J. Torregrosa, J.R. Serrano, F.J. Arnau, P. Piqueras, A fluid dynamic model for unsteady compressible flow in wall-flow diesel particulate filters, Energy 36 (2011) 671-684.

[35] H.P. Helfrich, D. Zwick, A trust region algorithm for parametric curve and surface fitting, Journal of Computational and Applied Mathematics 73 (1996) 119- 134.

[36] D.W. Marquardt, An algorithm for least-squares estimation of nonlinear parameters, Journal of the Society for Industrial and Applied Mathematics 11(2) (1963) 431-441.

[37] M.A. Luersen, R. Le Riche, Globalized Nelder-Mead method for engineering optimization, Computers and Structures 82 (2004) $2251-2260$.

\section{List of Tables}

- Table 1.- Macro-and meso-geometry of the characterized EATS.

\section{List of Figures}

- Figure 1.- Flowchart of the proposed methodology. Steps to define the transfer matrix in operating point (p) from baseline characterisation.

- Figure 2.- Schematic setup of the impulsive test rig: measurement of generated pressure pulse, composed pressure pulse at device inlet and transmitted pressure pulse at device outlet.

- Figure 3.- Example of pressure wave decomposition in (a) direct and (b) inverse tests. Pressure pulse 150 mbar in amplitude and $14 \mathrm{~ms}$ in duration in DOC+DPF-1. 
- Figure 4.- Comparison between experimental and parameterized transmission matrix coefficients of DOC+DPF-2 corresponding to a direct test $\left(r_{1}\right.$ and $\left.t_{12}\right)$ with a pressure pulse of $150 \mathrm{mbar}$ in amplitude and $14 \mathrm{~ms}$ in duration. Fitting based on one-step Fourier series parameterization.

- Figure 5.- Comparison between experimental and parameterized transmission matrix coefficients of SCR-1 corresponding to a direct test $\left(r_{1}\right.$ and $\left.t_{12}\right)$ with a pressure pulse of $150 \mathrm{mbar}$ in amplitude and $14 \mathrm{~ms}$ in duration. Fitting based on one-step and two-steps Fourier series parameterization.

- Figure 6.- Fitting of the transmission matrix coefficients of DOC+DPF-1 corresponding to inverse test $\left(r_{2}\right.$ and $\left.t_{21}\right)$ with change in excitation amplitude from 150 mbar (baseline) to 230 mbar, keeping Fourier series constants from the baseline point and tuning the characteristic period.

- Figure 7.- Fitting of the transmission matrix coefficients of DOC+DPF-1 corresponding to inverse test $\left(r_{2}\right.$ and $\left.t_{21}\right)$ with change in superimposed mean mass flow from $0 \mathrm{~kg} / \mathrm{h}$ (baseline) to $170 \mathrm{~kg} / \mathrm{h}$ (Mach 0.055), keeping Fourier series constants from the baseline point and tuning the characteristic period.

- Figure 8.- Decomposition into first-step Fourier series and deviation function of the 2-steps fitting of transmission matrix coefficients of SCR-1 corresponding to a direct test $\left(r_{1}\right.$ and $\left.t_{12}\right)$ with a pressure pulse of 150 mbar in amplitude and $14 \mathrm{~ms}$ in duration.

- Figure 9.- Error $\left(e_{f}\right)$ between experimental data and two-steps fitting as a function of the characteristic period. Pressure pulse 80 mbar in amplitude and $11 \mathrm{~ms}$ in duration for inverse test in SCR-1.

- Figure 10.- Influence of the frequency steps defining the experimental transmission matrix coefficients on the fitting accuracy. Pressure pulse 80 mbar in amplitude and $11 \mathrm{~ms}$ in duration for inverse test in SCR-1.

- Figure 11.- Influence of the selected maximum frequency on the fitting accuracy. Pressure pulse 150 mbar in amplitude and $14 \mathrm{~ms}$ in duration for inverse test in DOC+DPF-1.

- Figure 12.- Time domain modelling of TWC-1 with two different gas dynamic model setups of direct and inverse tests and excitations of different amplitude. (a) Direct test with 150 mbar in amplitude, (b) Direct test with 230 mbar in amplitude, (c) Inverse test with 150 mbar in amplitude, (d) Inverse test with 230 mbar in amplitude.

- Figure 13.- Comparison between the experimental and modelled real part of the transmission matrix coefficients corresponding to direct and inverse tests in TWC-1 with excitations of different amplitude. (a) Experimental reflection, (b) Experimental transmission, (c) Modelled reflection, (d) Modelled transmission.

- Figure 14.- Prediction of the transmission matrix coefficients of TWC-1 applying the phenomenological methodology to two gas dynamic computations corresponding to setups of different accuracy in the time domain. Pressure pulse 230 mbar in amplitude and $18 \mathrm{~ms}$ in duration in direct test.

- Figure 15.- Prediction of the transmission matrix coefficients of DOC+DPF-2 applying the phenomenological methodology. Pressure pulse $230 \mathrm{mbar}$ in amplitude and $18 \mathrm{~ms}$ in duration in direct test.

- Figure 16.- Prediction of the transfer matrix of TWC-1 applying the phenomenological methodology and comparison against experimental data and fitting results based on two-steps Fourier series parameterization. Pressure pulse of $230 \mathrm{mbar}$ in amplitude and $18 \mathrm{~ms}$ in duration. (a) $\mathrm{T}_{11}$, (b) $\mathrm{T}_{21}$, (c) $\mathrm{T}_{12}$, (d) $\mathrm{T}_{22}$. 
- Figure 17.- Prediction of the (a) direct and (b) inverse transmission loss of SCR-1 applying the phenomenological methodology and comparison against experimental data. Pressure pulse of $80 \mathrm{mbar}$ in amplitude and $11 \mathrm{~ms}$ in duration.

- Figure 18.- Prediction of the transfer matrix of TWC-1 applying the phenomenological methodology and comparison against experimental data and fitting results based on two-steps Fourier series parameterization. Pressure pulse of 120 mbar in amplitude and $12 \mathrm{~ms}$ in duration with a superimposed mean mass flow of $100 \mathrm{~kg} / \mathrm{h}$ (Mach 0.048). (a) $\mathrm{T}_{11}$, (b) $\mathrm{T}_{21}$, (c) $\mathrm{T}_{12}$, (d) $\mathrm{T}_{22}$.

- Figure 19.- Prediction of the (a) direct and (b) inverse transmission loss of TWC-1 applying the phenomenological methodology and comparison against experimental data. Pressure pulse of 120 mbar in amplitude and $12 \mathrm{~ms}$ in duration with a superimposed mean mass flow of $100 \mathrm{~kg} / \mathrm{h}$ (Mach 0.048). 


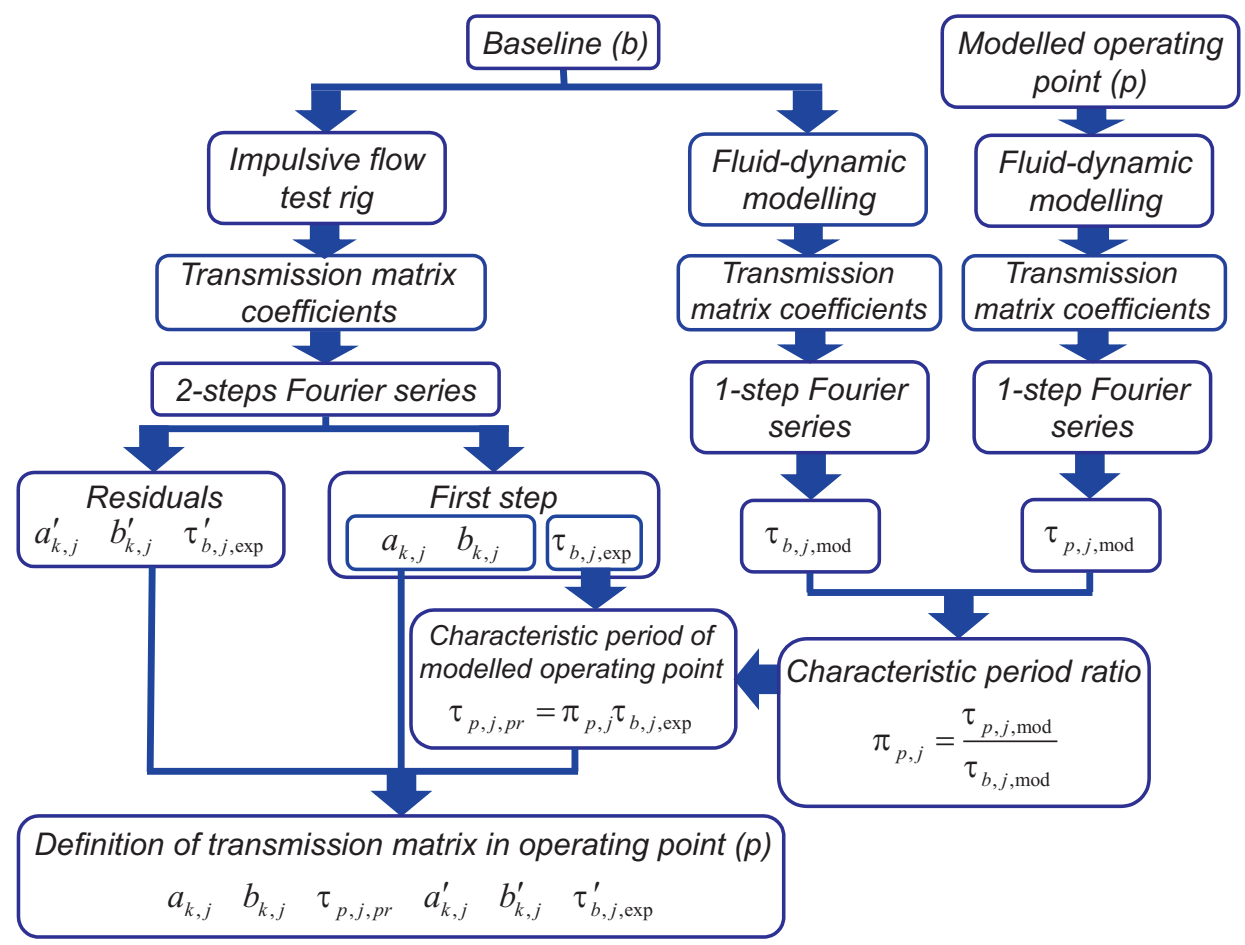

Figure 1: Flowchart of the proposed methodology. Steps to define the transfer matrix in operating point (p) from baseline characterisation.

Table 1: Macro-and meso-geometry of the characterized EATS.

\begin{tabular}{cccccccc}
\hline \hline & & DOC-1 & DPF-1 & DOC-2 & DPF-2 & TWC-1 & SCR-1 \\
\hline \hline$D$ & {$[\mathrm{~mm}]$} & 172 & 172 & 144 & 144 & 112 & 121 \\
$L$ & {$[\mathrm{~mm}]$} & 82 & 105 & 114 & 130 & 127 & 280 \\
$\alpha$ & {$[\mathrm{mm}]$} & 0.83 & 1.4 & 0.87 & 1.4 & 0.97 & 1.16 \\
$w_{w}$ & {$[\mathrm{~mm}]$} & 0.44 & 0.4 & 0.4 & 0.4 & 0.11 & 0.11 \\
$\sigma$ & {$[\mathrm{cpsi}]$} & 400 & 200 & 400 & 200 & 600 & 400 \\
\hline \hline
\end{tabular}




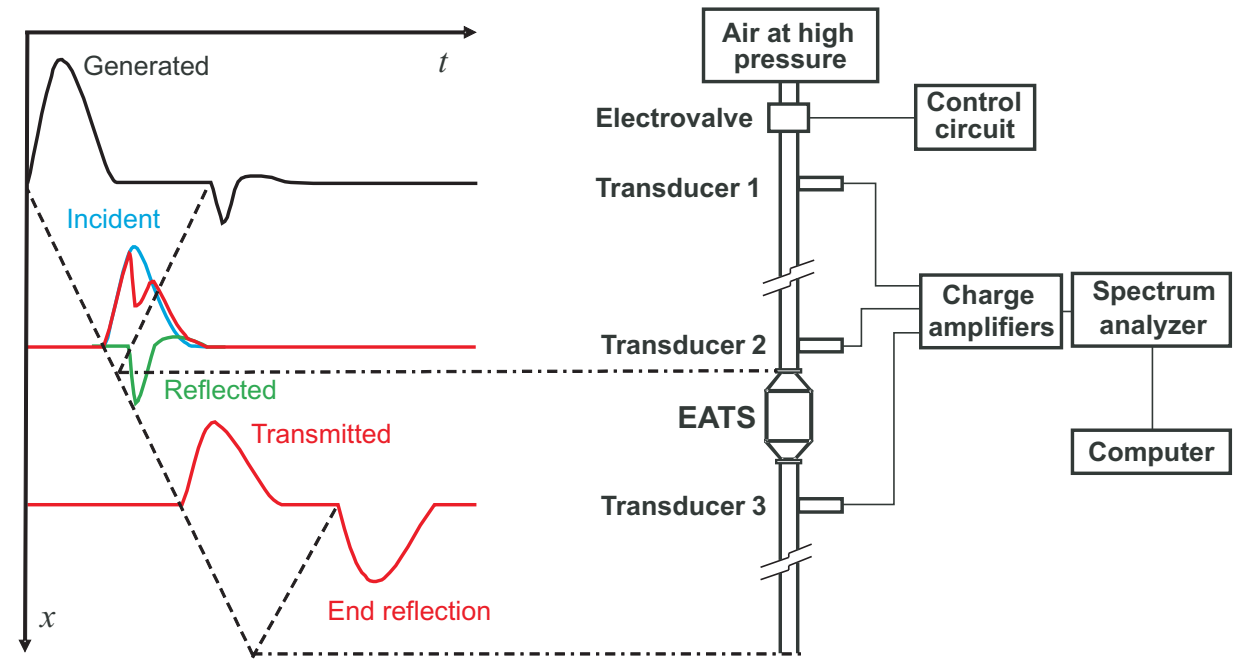

Figure 2: Schematic setup of the impulsive test rig: measurement of generated pressure pulse, composed pressure pulse at device inlet and transmitted pressure pulse at device outlet.

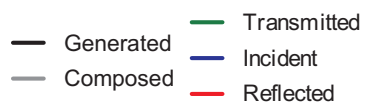

b) Inverse test
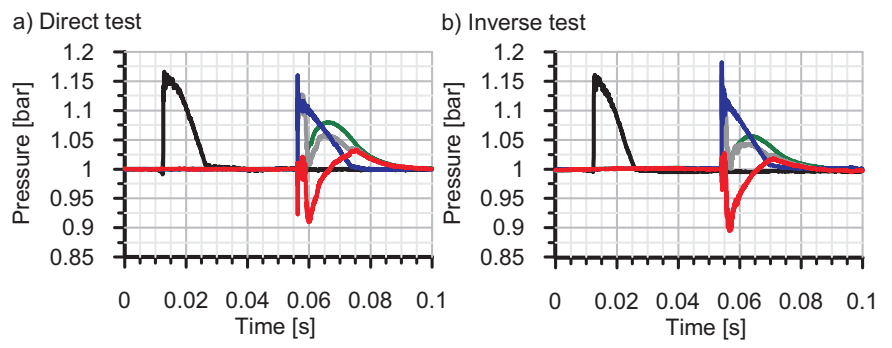

Figure 3: Example of pressure wave decomposition in (a) direct and (b) inverse tests. Pressure pulse $150 \mathrm{mbar}$ in amplitude and $14 \mathrm{~ms}$ in duration in DOC+DPF-1. 

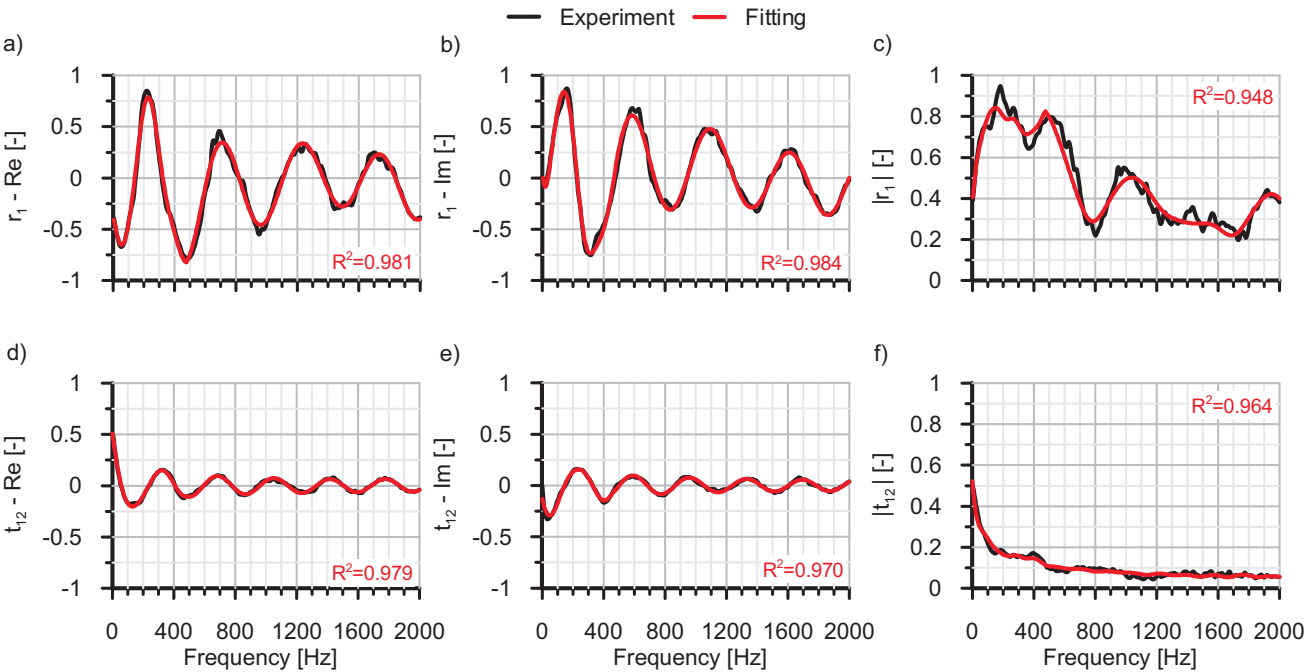

Figure 4: Comparison between experimental and parameterized transmission matrix coefficients of DOC+DPF-2 corresponding to a direct test $\left(r_{1}\right.$ and $t_{12}$ ) with a pressure pulse of $150 \mathrm{mbar}$ in amplitude and $14 \mathrm{~ms}$ in duration. Fitting based on one-step Fourier series parameterization.
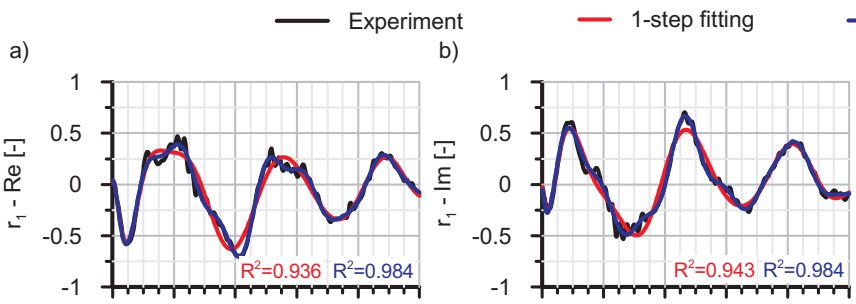

d)

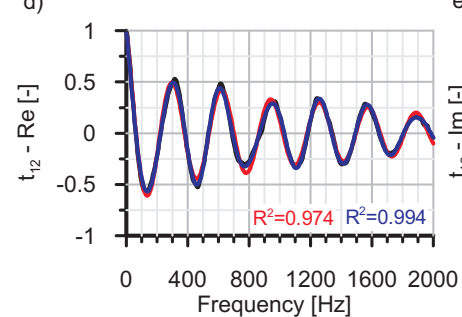

e)

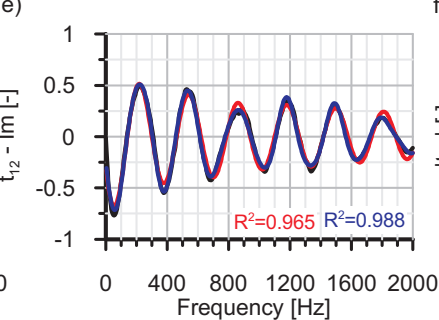

- 2-steps fitting

c)

Figure 5: Comparison between experimental and parameterized transmission matrix coefficients of SCR-1 corresponding to a direct test $\left(r_{1}\right.$ and $t_{12}$ ) with a pressure pulse of $150 \mathrm{mbar}$ in amplitude and $14 \mathrm{~ms}$ in duration. Fitting based on one-step and two-steps Fourier series parameterization. 

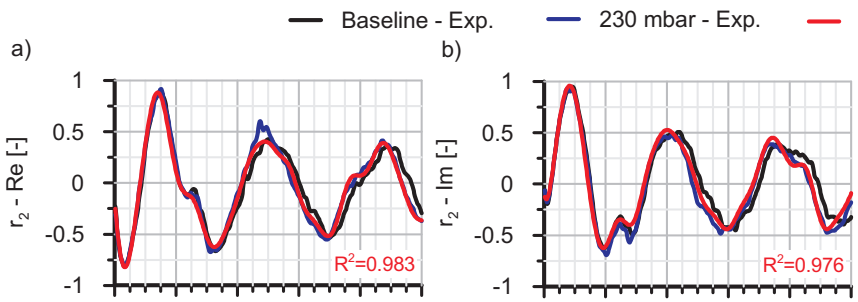

mbar - Fitting

c)

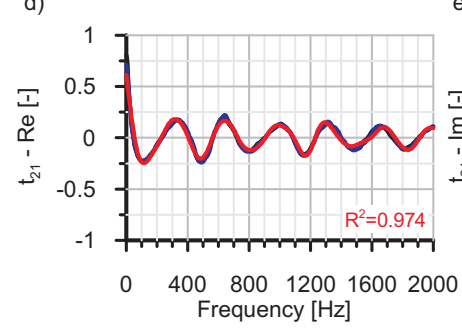

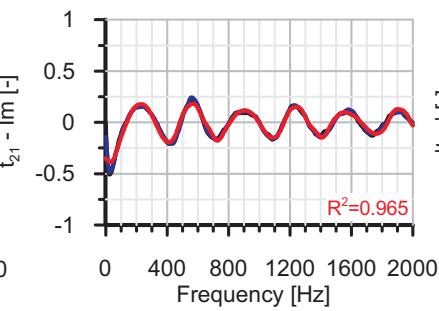
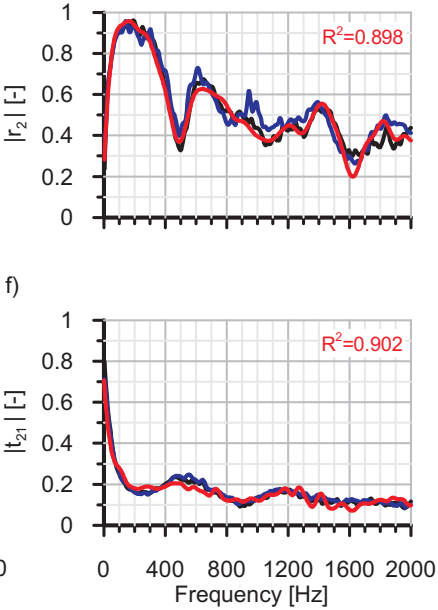

Figure 6: Fitting of the transmission matrix coefficients of DOC+DPF-1 corresponding to inverse test $\left(r_{2}\right.$ and $\left.t_{21}\right)$ with change in excitation amplitude from 150 mbar (baseline) to 230 mbar, keeping Fourier series constants from the baseline point and tuning the characteristic period.

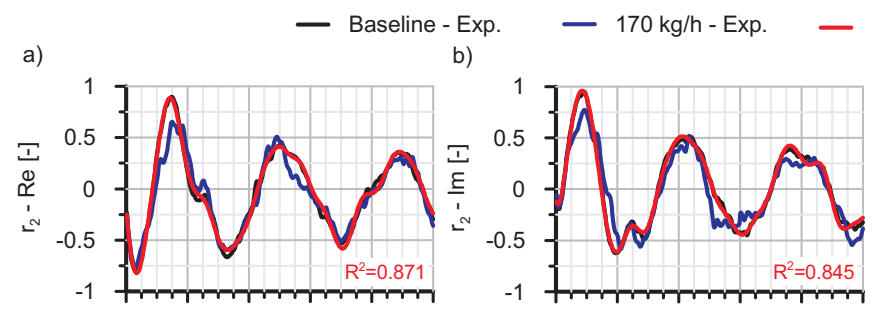

170 kg/h - Fitting

d)

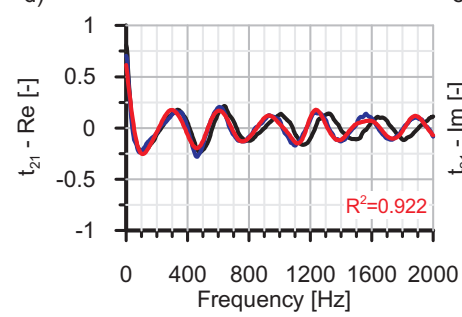

e)

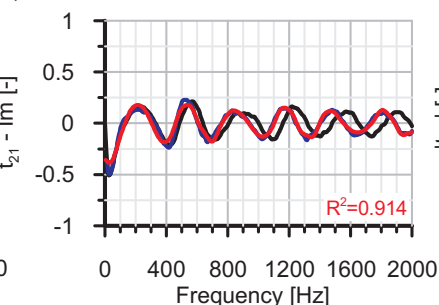

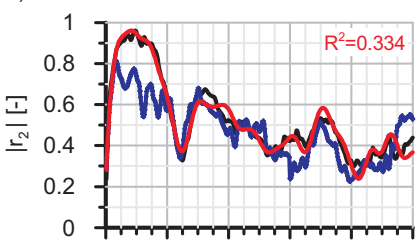

f)

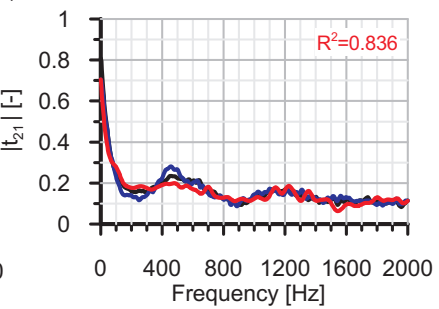

Figure 7: Fitting of the transmission matrix coefficients of DOC+DPF-1 corresponding to inverse test $\left(r_{2}\right.$ and $\left.t_{21}\right)$ with change in superimposed mean mass flow from $0 \mathrm{~kg} / \mathrm{h}$ (baseline) to $170 \mathrm{~kg} / \mathrm{h}$ (Mach 0.055 ), keeping Fourier series constants from the baseline point and tuning the characteristic period. 

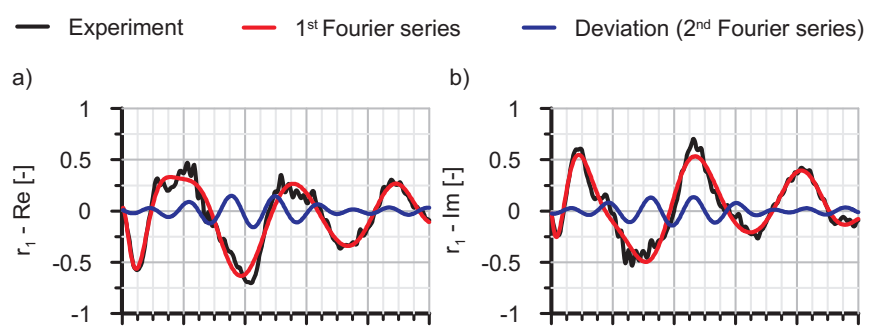

b)
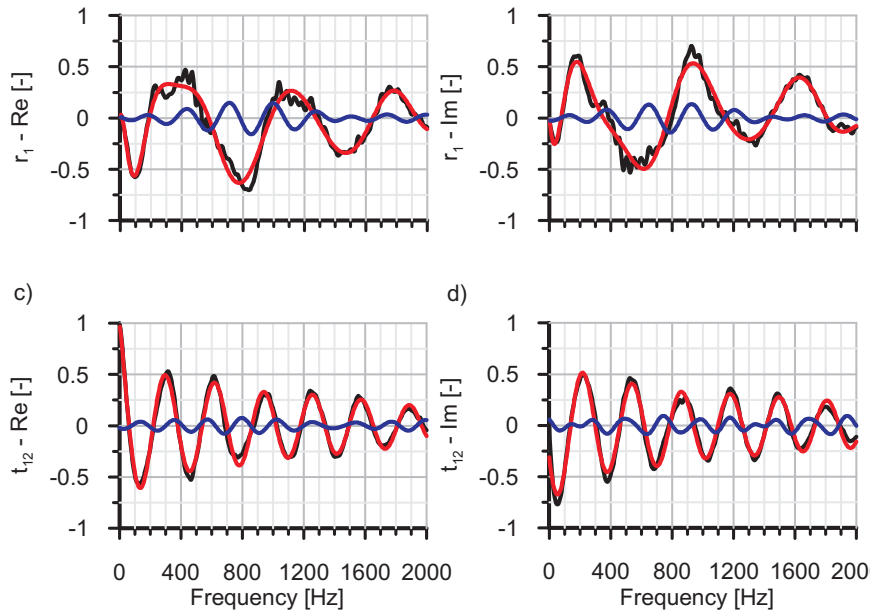

d)

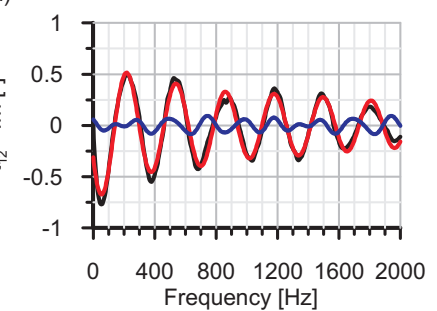

Figure 8: Decomposition into first-step Fourier series and deviation function of the 2-steps fitting of transmission matrix coefficients of SCR-1 corresponding to a direct test $\left(r_{1}\right.$ and $\left.t_{12}\right)$ with a pressure pulse of 150 mbar in amplitude and 14 ms in duration.
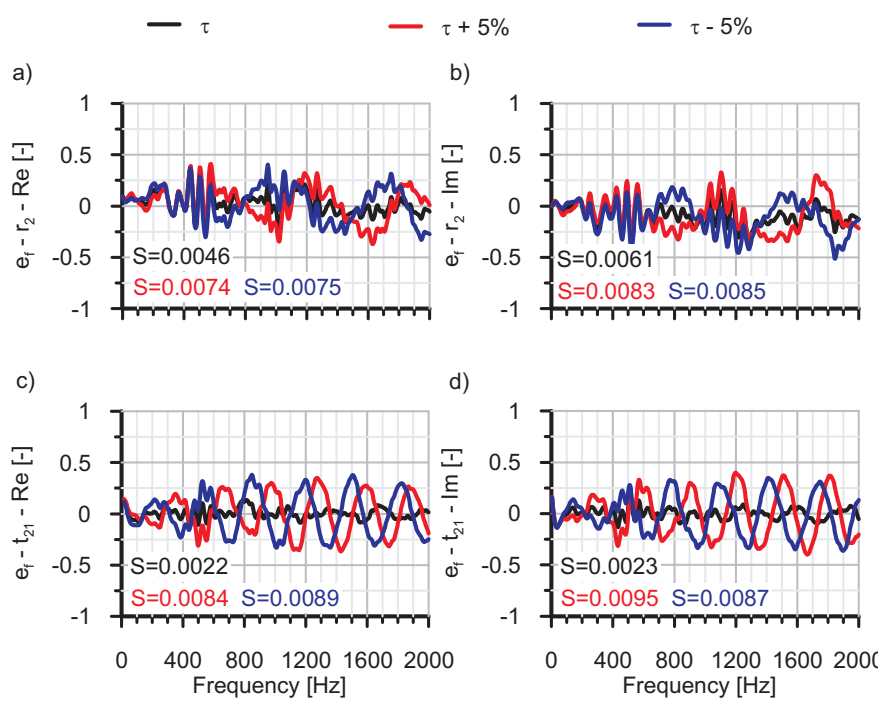

d)

Figure 9: Error $\left(e_{f}\right)$ between experimental data and two-steps fitting as a function of the characteristic period. Pressure pulse 80 mbar in amplitude and $11 \mathrm{~ms}$ in duration for inverse test in SCR-1. 


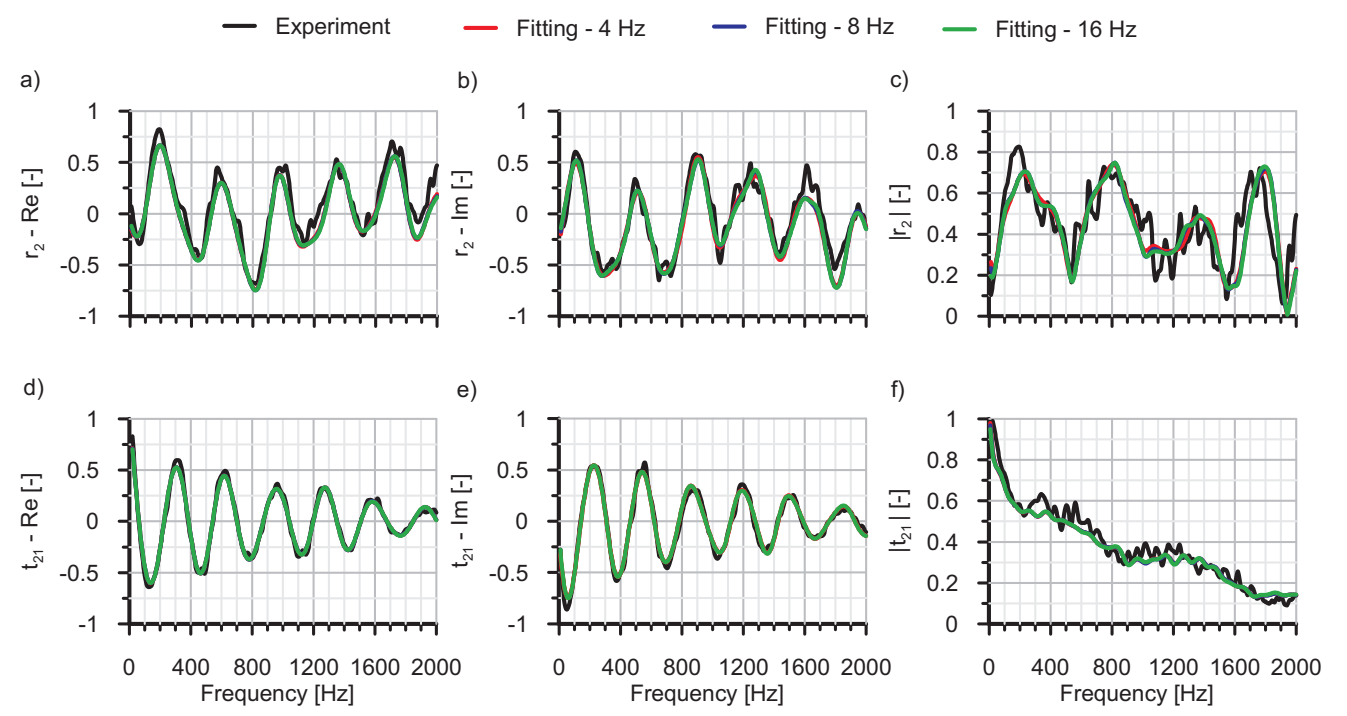

Figure 10: Influence of the frequency steps defining the experimental transmission matrix coefficients on the fitting accuracy. Pressure pulse $80 \mathrm{mbar}$ in amplitude and $11 \mathrm{~ms}$ in duration for inverse test in SCR-1.
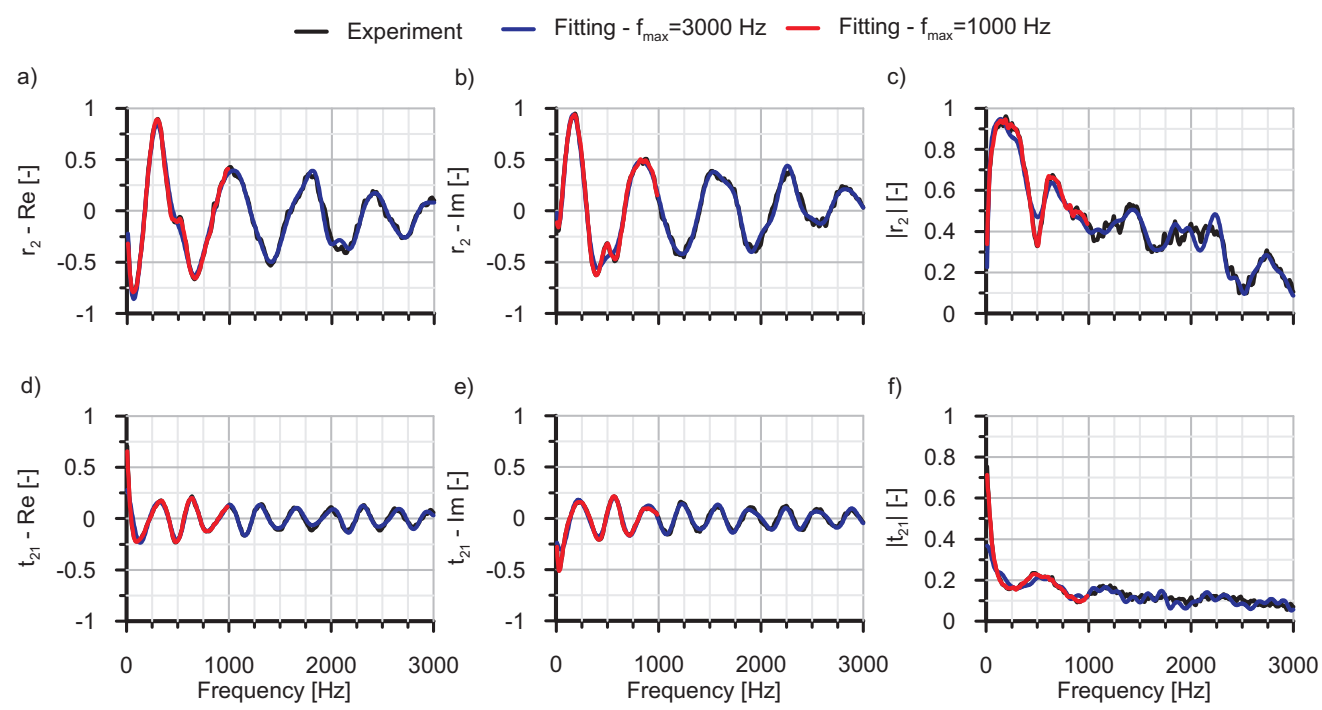

Figure 11: Influence of the selected maximum frequency on the fitting accuracy. Pressure pulse 150 mbar in amplitude and $14 \mathrm{~ms}$ in duration for inverse test in DOC+DPF-1. 

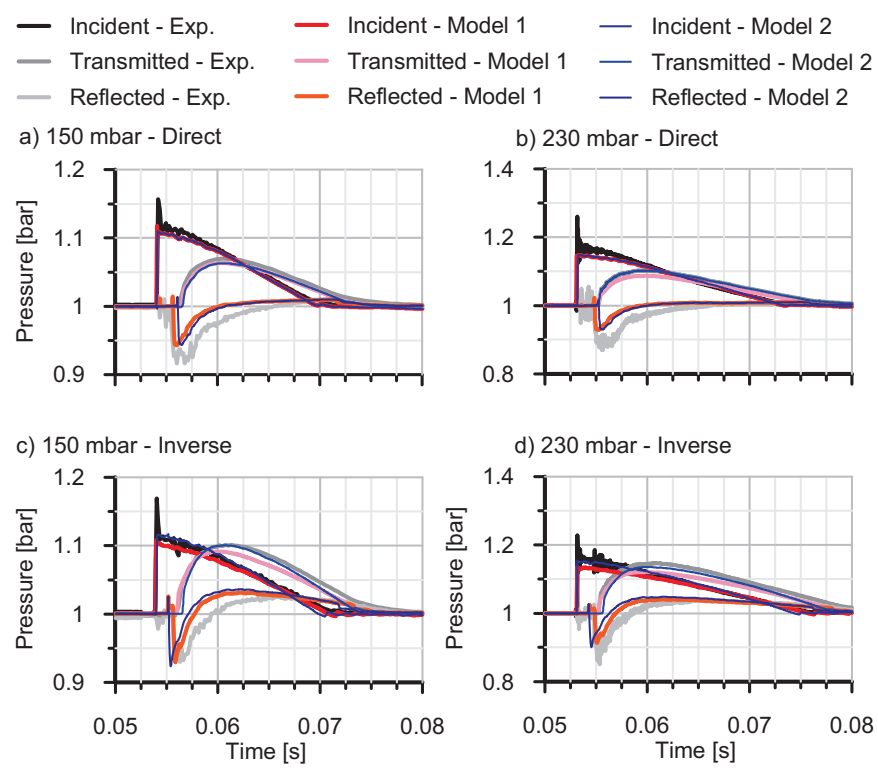

Figure 12: Time domain modelling of TWC-1 with two different gas dynamic model setups of direct and inverse tests and excitations of different amplitude. (a) Direct test with 150 mbar in amplitude, (b) Direct test with 230 mbar in amplitude, (c) Inverse test with 150 mbar in amplitude, (d) Inverse test with 230 mbar in amplitude.
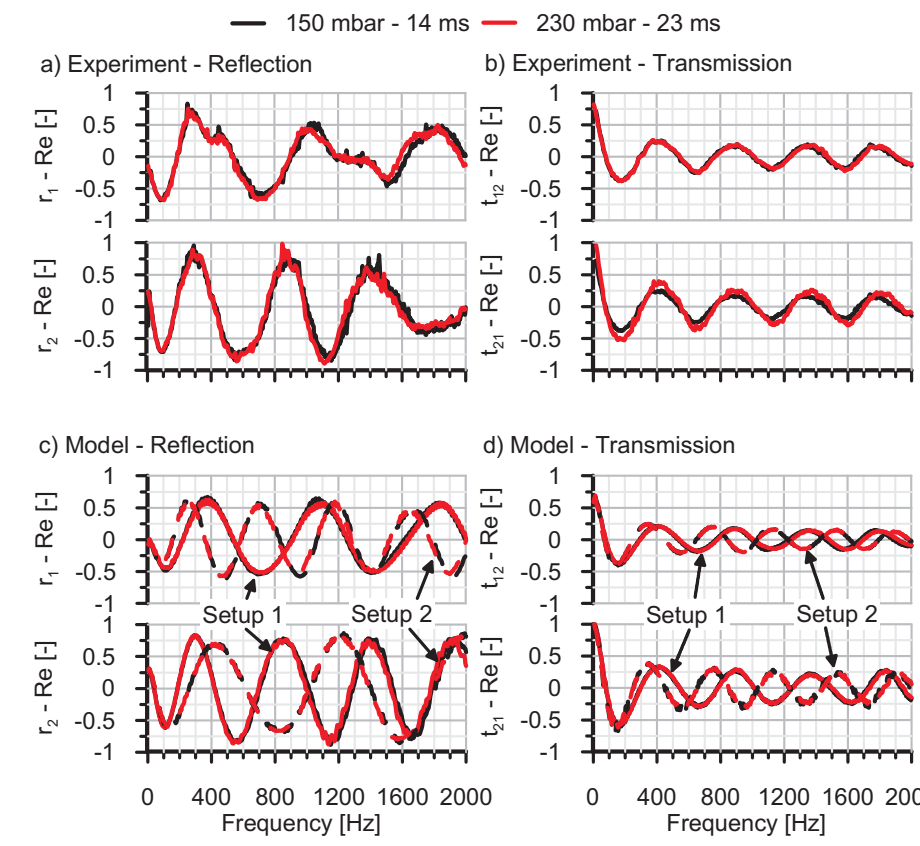

d) Model - Transmission

Figure 13: Comparison between the experimental and modelled real part of the transmission matrix coefficients corresponding to direct and inverse tests in TWC-1 with excitations of different amplitude. (a) Experimental reflection, (b) Experimental transmission, (c) Modelled reflection, (d) Modelled transmission. 


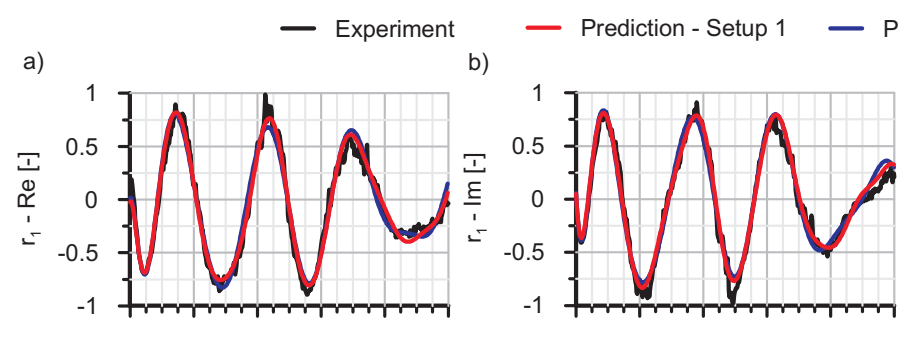

Prediction - Setup 2

d)

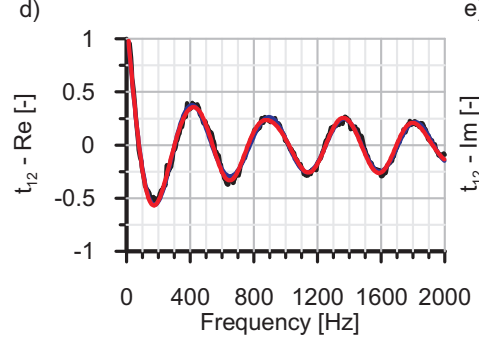

e)

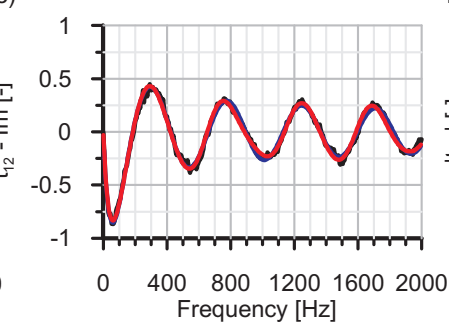

c)

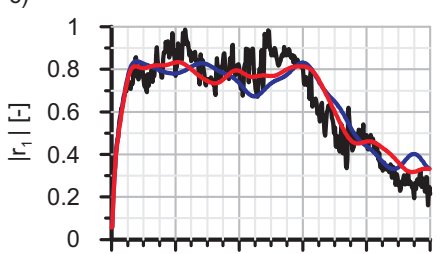

f)

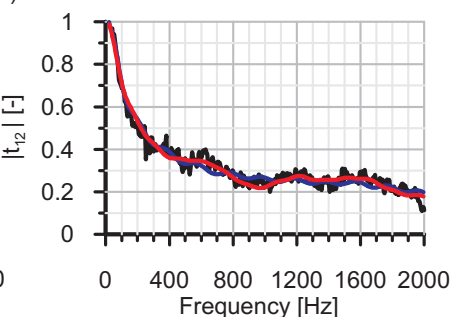

Figure 14: Prediction of the transmission matrix coefficients of TWC-1 applying the phenomenological methodology to two gas dynamic computations corresponding to setups of different accuracy in the time domain. Pressure pulse 230 mbar in amplitude and $18 \mathrm{~ms}$ in duration in direct test.
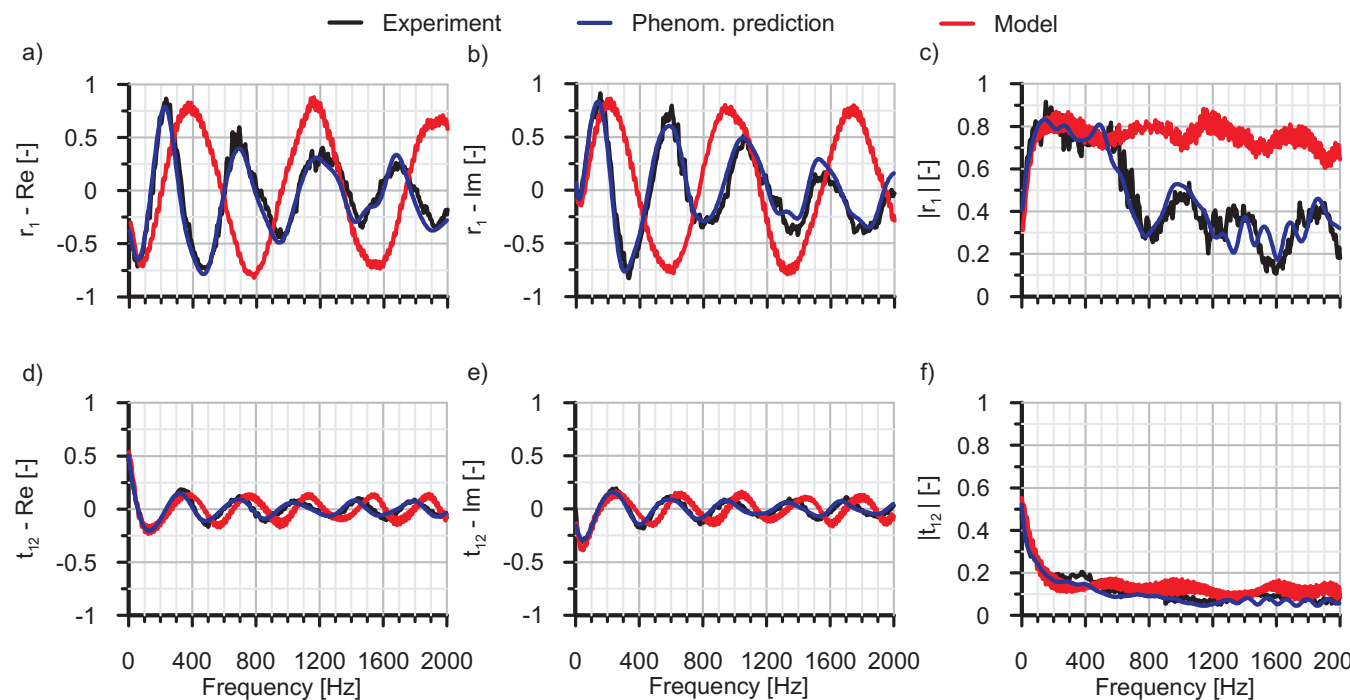

e)
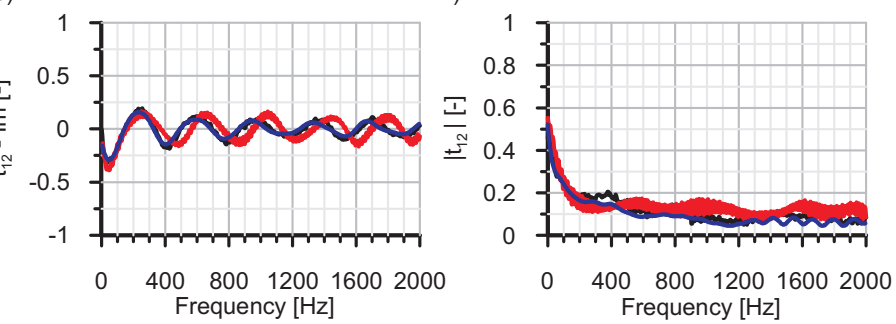

Figure 15: Prediction of the transmission matrix coefficients of DOC+DPF-2 applying the phenomenological methodology. Pressure pulse $230 \mathrm{mbar}$ in amplitude and $18 \mathrm{~ms}$ in duration in direct test. 

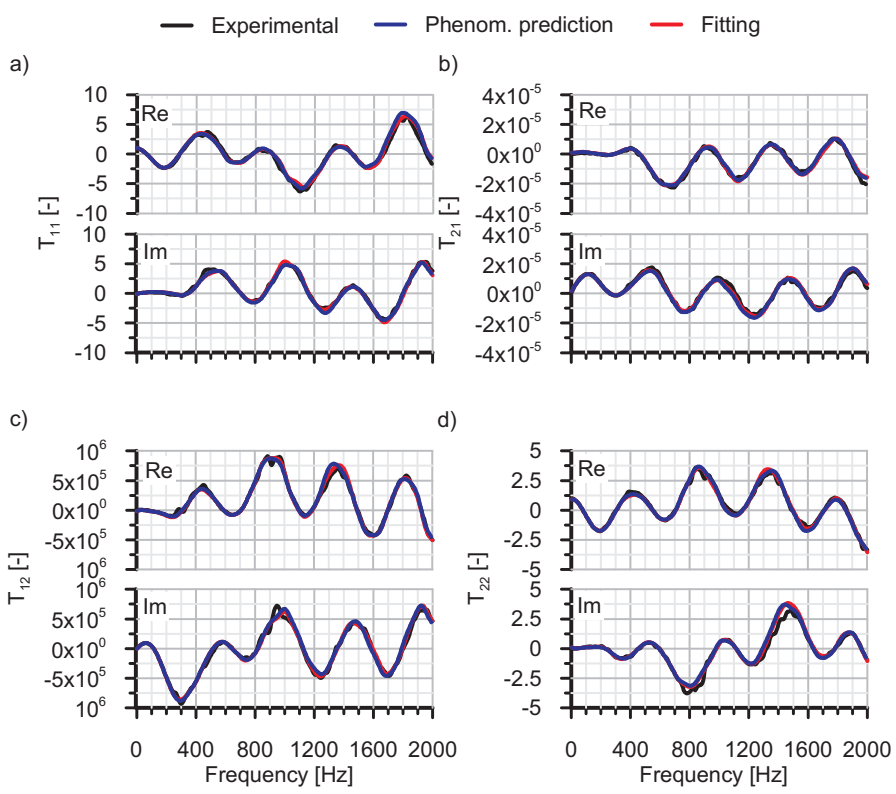

Figure 16: Prediction of the transfer matrix of TWC-1 applying the phenomenological methodology and comparison against experimental data and fitting results based on two-steps Fourier series parameterization. Pressure pulse of $230 \mathrm{mbar}$ in amplitude and $18 \mathrm{~ms}$ in duration. (a) $\mathrm{T}_{11}$, (b) $\mathrm{T}_{21}$, (c) $\mathrm{T}_{12}$, (d) $\mathrm{T}_{22}$.

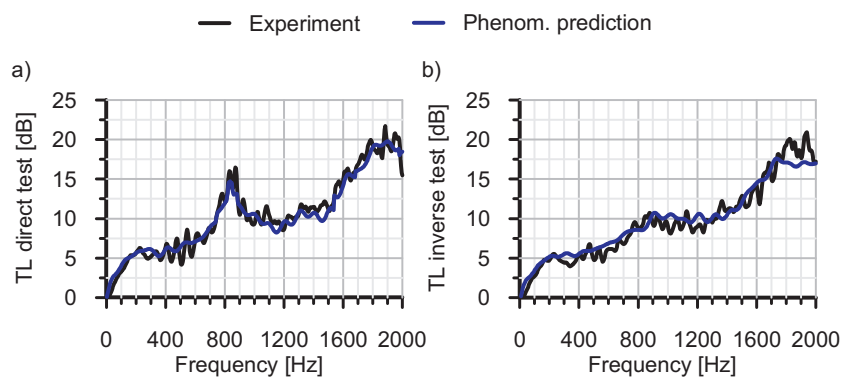

Figure 17: Prediction of the (a) direct and (b) inverse transmission loss of SCR-1 applying the phenomenological methodology and comparison against experimental data. Pressure pulse of 80 mbar in amplitude and $11 \mathrm{~ms}$ in duration. 


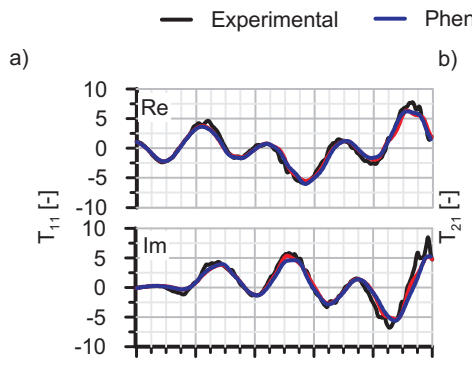

b)

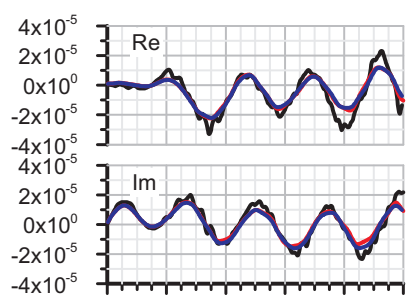

c)

d)
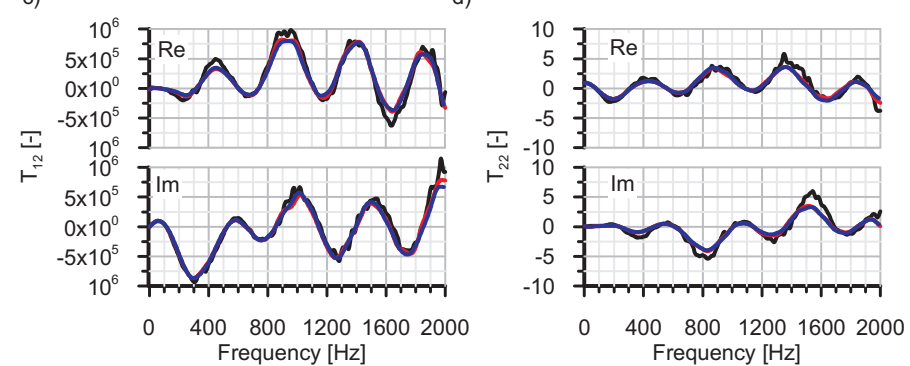

Figure 18: Prediction of the transfer matrix of TWC-1 applying the phenomenological methodology and comparison against experimental data and fitting results based on two-steps Fourier series parameterization. Pressure pulse of 120 mbar in amplitude and 12 ms in duration with a superimposed mean mass flow of $100 \mathrm{~kg} / \mathrm{h}$ (Mach 0.048). (a) $\mathrm{T}_{11}$, (b) $\mathrm{T}_{21}$, (c) $\mathrm{T}_{12}$, (d) $\mathrm{T}_{22}$.

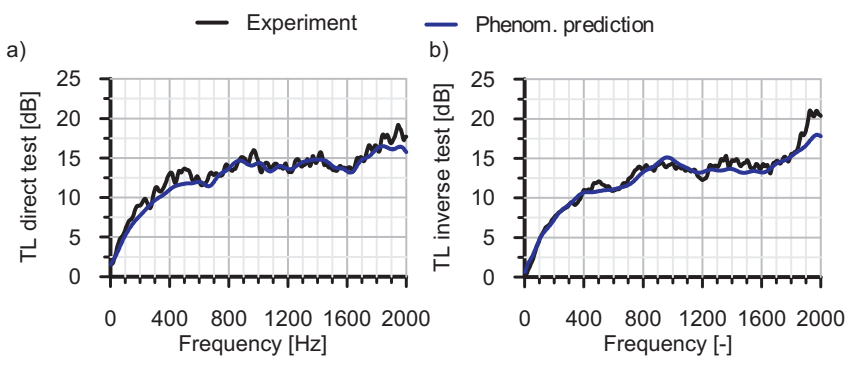

Figure 19: Prediction of the (a) direct and (b) inverse transmission loss of TWC-1 applying the phenomenological methodology and comparison against experimental data. Pressure pulse of $120 \mathrm{mbar}$ in amplitude and $12 \mathrm{~ms}$ in duration with a superimposed mean mass flow of $100 \mathrm{~kg} / \mathrm{h}$ (Mach 0.048). 